\title{
A Mathematical Model of Cancer Stem Cell Lineage Population Dynamics with Mutation Accumulation and Telomere Length Hierarchies
}

\author{
G. Kapitanov * \\ Vanderbilt University Department of Mathematics \\ 1326 Stevenson Center, Nashville, TN 37240, USA
}

\begin{abstract}
There is evidence that cancer develops when cells acquire a sequence of mutations that alter normal cell characteristics. This sequence determines a hierarchy among the cells, based on how many more mutations they need to accumulate in order to become cancerous. When cells divide, they exhibit telomere loss and differentiate, which defines another cell hierarchy, on top of which is the stem cell. We propose a mutation-generation model, which combines the mutation-accumulation hierarchy with the differentiation hierarchy of the cells, allowing us to take a step further in examining cancer development and growth. The results of the model support the hypothesis of the cancer stem cell's role in cancer pathogenesis: a very small fraction of the cancer cell population is responsible for the cancer growth and development. Also, according to the model, the nature of mutation accumulation is sufficient to explain the faster growth of the cancer cell population. However, numerical results show that in order for a cancer to develop within a reasonable time frame, cancer cells need to exhibit a higher proliferation rate than normal cells.
\end{abstract}

Key words: cancer, stem cells, cancer stem cell, mathematical model, mutation, telomere shortening

AMS subject classification: 92C99, 92D15

*E-mail: georgi.i.kapitanov@ vanderbilt.edu 


\section{Introduction}

Telomeres are the ends of chromosomes in eukaryotic cells. They are repeated DNA sequences that do not contain genetic information. A cell goes through several phases in its cycle, the final being mitosis - division of the cell into two daughters. Although both daughters are genetically identical to the mother, the splitting of chromosomes is not symmetrical - there is DNA loss. This loss occurs at the ends of the chromosomes, so it is only loss of telomeres. Each cell has a finite number of telomeres, therefore its descendants can only divide a finite number of times (the cells that cannot divide any further die eventually). The number of those divisions is called a Hayflick limit [29][40][32].

At the top of the telomere hierarchy is the stem cell. The stem cell has two properties that characterize it: self-renewal and potency. Self-renewal is the ability of the cell to divide and reproduce itself without differentiation (i.e. one daughter of the stem cell remains a stem cell). Potency is the ability of the stem cell to differentiate into specialized cell types (i.e. the other daughter will be a cell with more specific functions). Those two abilities allow the stem cells to create organs and tissues: self-renewal guarantees constant supply of cells, and differentiation guarantees the production of cells whose functions are essential for the tissue[40][32]. Other types of cells in the differentiation hierarchy are progenitor cells, and differentiated (mature) cells. Differentiated cells perform very specific functions in the body. They cannot self-renew. Progenitor cells are a middle stage between stem cells and differentiated cells.

A hierarchy imposed in the model divides cells into classes, based on how long their telomeres are, which coincides with how differentiated they are. The more differentiated cells have the shortest and the least differentiated cells, the stem cells, have the longest telomeres.

There is strong evidence that cancer develops after several stages of mutagenesis of normal cells (Figure 2). Mutations can not only change the characteristics and functions of the cell but also alter its capability of survival. Cancer cells generally proliferate faster, are more competitive for resources, and grow larger than normal cells[49][37][12].

In the present model a mutation can occur only during replication of DNA (i.e. during cell division). This mutation would be exhibited by the daughter cells. The daughter cells also inherit the mutations their mother cell has accumulated before division. The descendants of the daughter cells can acquire further mutations until, eventually, their descendants reach the number (and type) of mutations necessary for the formation of a cancer cell. Thus, we impose a hierarchial structure upon the cells, based on how many mutations they have accumulated (which is equivalent to how close they are to being cancerous). However, both non-cancer and cancer cells exhibit the telomere hierarchy. Therefore, combining both hierarchies, we can split cells into classes depending on the number of acquired mutations, and the number of differentiations left until their descendants become fully differentiated.

The main objective of the model presented in this article is to describe the interactions between cells of several levels of differentiation and malignancy by tracking their lineages. The model tries to address three questions: 
1. Considering cell mutation as a dynamic population process, rather than a one-time random event, what can we show about cancer cell population growth in relation to the growth of the populations of non-cancer cells?

2. What is the role of stem cells in the cell population dynamics?

3. Is the cancer stem cell count as small as scientists have claimed (some results claim that only one in ten thousand cancer cells is a cancer stem cell[13][9])?

The model addresses these questions through both mathematical analysis and numerical simulations and the answers appear in the Discussion.

Difficulty in observing and experimenting with stem cells has led to a rise in mathematical models, which can describe cell growth and interaction. Several previous models have considered a telomere or differentiation hierarchy[33][31]. In [2], Arino, Kimmel, and Webb looked at a model of telomere loss in both discrete and continuous time. Under their assumptions, the cells grew in polynomial time, the fastest growing cells being the ones with the least telomeres. However, it is the initial reservoir of the cells with highest amount of telomeres that determines the size of the asymptotic population. In [3], Arino, Sánchez, and Webb looked at telomere loss using a continuous model involving a linear system of partial differential equations. In fact, the model presented here can be considered an expansion of their model. Their result was consistent with [3] - polynomial growth of all cell classes and faster growth of cells with less telomeres. In [29], Levy, et al, gave another discrete model of telomere loss, exploring its connection to cell senescence. Discrete classification of maturity and tracking of a cell cohort during successive divisions have been done previously in [7] by Bernard, Pujo-Menjouet, and Mackey. These authors considered cells in two phases: resting phase and proliferative phase. Their results are consistent with experimental data and their model is a good framework for characterization of different cell subpopulations. In [22] Fearon and Vogelstein introduced a model for sequential accumulation of mutations, known as a "Vogelgram". Their hypothesis, that a cell goes through several mutations to become cancerous, is a very important assumption for our model. In [24], Gentry, Ashkenazi, and Jackson developed a model that combined cell hierarchy with sequential accumulation of mutations. They focused on the effect different types of mutations had on cancer growth. The mathematical model proposed in the present paper differs from theirs in several ways. In [24], the model considers cell maturity as a continuous variable correlated to differentiation, while in the present work differentiation is discrete and determines cell classes. The present model tracks cell lineages through multiple generations, not unlike [7], and focuses on the role of the stem cells as generators of this lineage.

\section{The Linear Model}

The biological assumptions of the model are:

1. Telomere loss only occurs during division. There is no loss of telomeres driven by outside sources (oxidation, etc.[1][46]). There is also no gain of telomeres - the model does not 


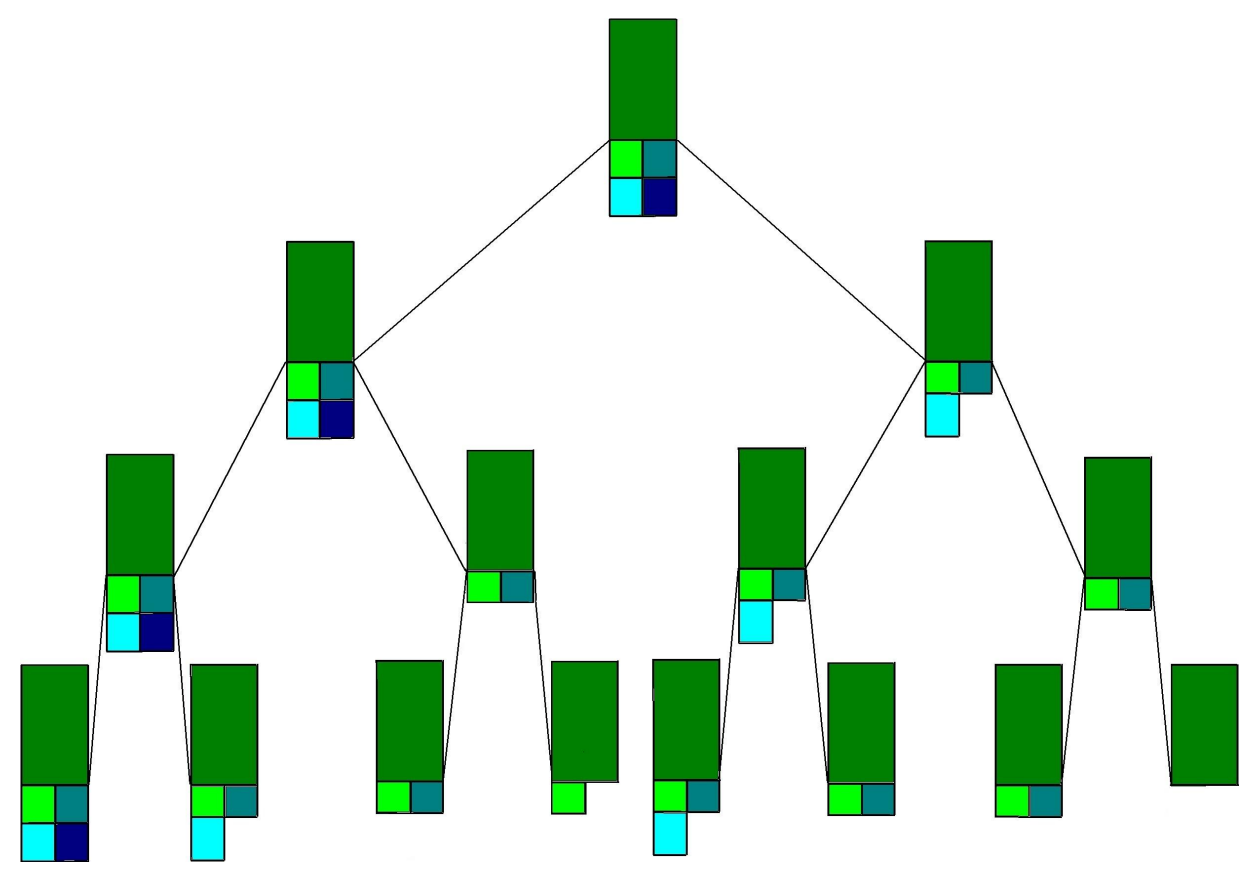

Figure 1: The process of asymmetrical telomere shortening as a cell divides. The top cell is a stem cell with full length chromosomes. The leftmost branch of division consists of stem cells (self-renewal). The other branches depict more differentiated cells. They exhibit loss of chromosome ends - telomeres [29]. Note that a cell may lose different telomere lengths when dividing.

address the role of the enzyme telomerase. These simplifications allow us to concentrate on the cell lineages and the mother-daughter cell relationship by omitting some complexities of the cell biology.

2. If a mutation occurs during the division of a cell, the mutation is exhibited by the daughters of the cell and not by the mother cell. Therefore, the model does not consider somatic mutations or the complex mechanisms through which the body attacks and destroys cells with a detected mutation [25]. This assumption is also made with the intention to allow the model to focus on the interaction of the cell lineages.

The model is formulated as the following system of partial differential equations:

$$
\begin{gathered}
\frac{\partial u_{j, i}(a, t)}{\partial t}+\frac{\partial u_{j, i}(a, t)}{\partial a}=-\left(\mu_{j, i}(a)+\beta_{j, i}(a)\right) u_{j, i}(a, t) \\
u_{j, i}(0, t)=2 \sum_{k=j}^{n}\left(p_{j, k, i} \int_{0}^{\infty} \beta_{k, i}(a) u_{k, i}(a, t) d a+q_{j, k, i-1} \int_{0}^{\infty} \beta_{k, i-1}(a) u_{k, i-1}(a, t) d a\right) \\
u_{j, i}(a, 0)=\phi_{j, i}(a)
\end{gathered}
$$

Note: 


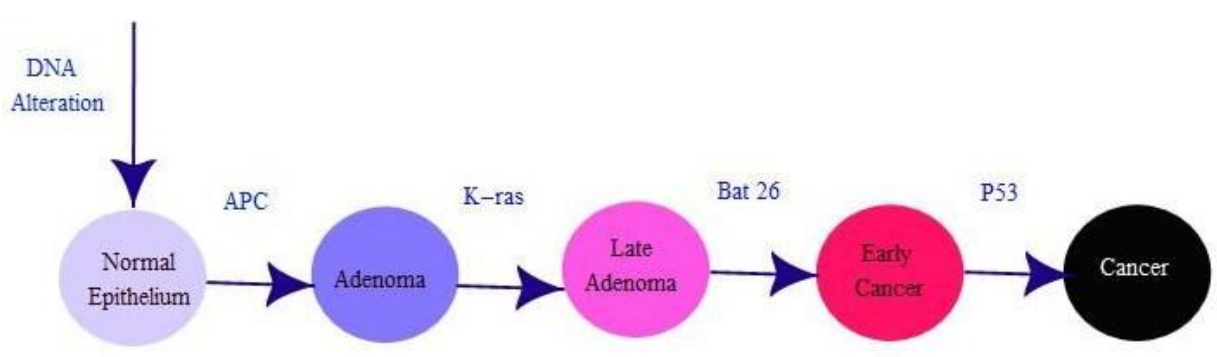

Figure 2: A Genetic Model for Colorectal Tumorigenesis. This is an example of a Vogelgram - multistep cancer progression model [22]

1. $j=1, \ldots, n$ represents the number of telomeres of a cell. Hence it also represents the number of divisions a cell has to go through until one of its descendants cannot divide any further. Moreover, it is the number of differentiation stages the lineage of a cell goes through until a fully differentiated cell is produced. $i=0, \ldots, m-1$ is the number of mutations a cell has accumulated. Therefore, $n$ is the number of differentiation classes and $m$ is the number of mutation classes. Stem cells are of class $j=n$ and differentiated cells are of class $j=1$. Normal cells have $i=0$ mutations and cancer cells have acquired $i=m-1$ mutations. Also, for the rest of the paper, let $N \stackrel{\text { def }}{=} n m$

2. For $t \geq 0, u_{j, i}(a, t) \in L_{1}([0, \infty))$, represents the density of cells with age $a$ at time $t$, in the $j^{\text {th }}$ telomere class, with $i$ mutations.

3. $\mu_{j, i}(a) \geq 0$, is the age-specific mortality rate of cells in the $j^{\text {th }}$ telomere, $i^{\text {th }}$ mutation class.

4. $\beta_{j, i}(a)>0$, is the age-specific proliferation rate of cells in the $j^{\text {th }}$ telomere, $i^{\text {th }}$ mutation class.

5. $p_{j, k, i}>0$, is the probability that one of the daughters of a cell in the $k^{\text {th }}$ telomere, $i^{\text {th }}$ mutation class will be a cell in the $j^{\text {th }}$ telomere, $i^{\text {th }}$ mutation class.

6. $q_{j, k, i-1}>0$, is the probability that a cell in the $k^{\text {th }}$ telomere, $(i-1)^{t h}$ mutation class will produce, by acquiring a mutation during division, a cell in the $j^{\text {th }}$ telomere, $i^{\text {th }}$ mutation class.

Hypothesis 2.1. A mother cell in the $j^{\text {th }}$ telomere, $i^{\text {th }}$ mutation class, $j=1, \ldots, n, i=0, \ldots, m-1$, produces one daughter cell in the same class. The other daughter cell could be either in a lower telomere, same mutation class, lower telomere, $(i+1)^{t h}$ mutation class, or same telomere, $(i+1)^{t h}$ mutation class. We do not keep track of cells in the $0^{\text {th }}$ telomere class (they cannot divide). A cell with $m-1$ mutations is considered cancerous and cannot acquire any further mutations.

The hypothesis means that: 
1. $p_{j, j, i}=\frac{1}{2}, \forall 1 \leq j \leq n, 0 \leq i \leq m-1$.

2. $p_{j, k, i}=0$ for $j>k, \forall 2 \leq j \leq n, 0 \leq i \leq m-1$.

3. $q_{j, k, i}=0$ for $j>k, \forall 2 \leq j \leq n, 0 \leq i \leq m-1$.

4. $\sum_{k=j+1}^{n} p_{j, k, i}+\sum_{k=j}^{n} q_{j, k, i}=\frac{1}{2}, \forall 1 \leq j \leq n ; 0 \leq i \leq m-2$.

Part 2 and 3 of the hypothesis simply make sure that a mother cell cannot give birth to daughters with more telomeres or less mutations. Part 1 assumes asymmetrical division of cells. The self-renewal property of the stem cell is consistent with the assumption of asymmetrical division [50][45]. For more differentiated cells, the asymmetry hypothesis is inconclusive and the telomere loss rate is not uniform [29]. Still, asymmetrical division has been shown in several eukaryotic species [35].

\section{Linear Model Analysis}

Hypothesis 3.1. $\mu_{j, i}(a)=\mu_{j, i} \geq 0$ and $\beta_{j, i}(a)=\beta_{j, i}>0, \forall 1 \leq j \leq n ; 0 \leq i \leq m-1$ (i.e. they are constants).

Integrating 2.1 with respect to $a$ over $[0, \infty)$, we get:

$$
\int_{0}^{\infty} \frac{\partial u_{j, i}(a, t)}{\partial t} d a-u_{j, i}(0, t)=-\left(\mu_{j, i}+\beta_{j, i}\right) \int_{0}^{\infty} u_{j, i}(a, t) d a
$$

Since $u_{j, i}(a, t)$ is a density function, $\int_{0}^{\infty} u_{j, i}(a, t) d a$ gives the total population of the $(j, i)^{t h}$ class of cells. Call it $U_{j, i}(t)$. Also,

$$
u_{j, i}(0, t)=2 \sum_{k=j}^{n}\left(p_{j, k, i} \int_{0}^{\infty} \beta_{k, i} u_{k, i}(a, t) d a+q_{j, k, i-1} \int_{0}^{\infty} \beta_{k, i-1} u_{k, i-1}(a, t) d a\right)
$$

Then the problem is recast as a system of ordinary differential equations:

$$
\begin{gathered}
U_{j, i}^{\prime}(t)=-\left(\mu_{j, i}+\beta_{j, i}\right) U_{j, i}(t)+2 \sum_{k=j}^{n}\left(p_{j, k, i} \beta_{k, i} U_{k, i}(t)+q_{j, k, i-1} \beta_{k, i-1} U_{k, i-1}(t)\right) \\
=-\left(\mu_{j, i}+\beta_{j, i}\right) U_{j, i}(t)+2 p_{j, j, i} \beta_{j, i} U_{j, i}(t)+ \\
+2 \sum_{k=j+1}^{n} p_{j, k, i} \beta_{k, i} U_{k, i}(t)+2 \sum_{k=j}^{n} q_{j, k, i-1} \beta_{k, i-1} U_{k, i-1}(t)
\end{gathered}
$$

From Hypotheses 2.1, since $p_{j, j, i}=\frac{1}{2}$, Equation 3.2 simplifies to:

$$
U_{j, i}^{\prime}(t)=-\mu_{j, i} U_{j, i}(t)+2 \sum_{k=j+1}^{n} p_{j, k, i} \beta_{k, i} U_{k, i}(t)+2 \sum_{k=j}^{n} q_{j, k, i-1} \beta_{k, i-1} U_{k, i-1}(t)
$$


Let $\vec{U}(t)=\left(U_{1,0}(t), \ldots, U_{n, 0}(t), U_{1,1}(t), \ldots, U_{n, 1}(t), \ldots, U_{j, i}(t), \ldots, U_{n, m-1}(t)\right)^{T}$. Then, the system of equations can be rewritten as:

$$
\begin{gathered}
\vec{U}^{\prime}(t)=A \vec{U}(t) \\
\vec{U}(0)=\vec{\Phi}
\end{gathered}
$$

where:

1. $A$ is an $N \times N$ matrix that can be described as an $m \times m$ block matrix. Each block is an $n \times n$ matrix. $A$ is a lower-triangular block matrix.

2. The blocks that form $A$ are of three types:

(a) 0 blocks $(n \times n 0$ matrix $)$

(b) $P_{i}$ blocks (they occupy the main block-diagonal of $A$ ): $n \times n$ upper-triangular matrices that describe the proliferation dynamics within the $i^{\text {th }}$ mutation class of cells. The diagonal of a $P_{i}$ matrix consists of the mortality rates $-\mu_{j, i}, 1 \leq j \leq n$, where the $j$ corresponds to the telomere class of the cells whose mortality $\mu_{j, i}$ describes and also the row $-\mu_{j, i}$ occupies in $P_{i} . P_{i}$ is an upper-triangular matrix, so its other non-zero elements (all are strictly positive) are located above the main diagonal and consist of the rates $2 p_{j, k, i} \beta_{k, i}$ with which cells in class $(k, i)$ produce cells in class $(j, i)(1 \leq j \leq$ $n-1 ; j+1 \leq k \leq n ; 0 \leq i \leq m-1)$ during mitosis. $2 p_{j, k, i} \beta_{k, i}$ is the $(j, k)^{t h}$ element of $P_{i}$.

(c) $Q_{i}$ blocks (they occupy the first block subdiagonal of $A$ ): $n \times n$ upper-triangular matrices that describe the mutation dynamics between the $(i-1)^{t h}$ and the $i^{\text {th }}$ mutation classes (the cells of mutation class $i-1$ mutate into cells of class $i, 1 \leq i \leq m-1$ ). Its elements are the rates $2 q_{j, k, i-1} \beta_{k, i-1}$ with which cells of class $(k, i-1)$ produce cells of class $(j, i)$ during mitosis $\left(1 \leq j \leq n-1 ; j \leq k \leq n ; 1 \leq i \leq m-1.2 q_{j, k, i-1} \beta_{k, i-1}\right.$ is the $(j, k)^{t h}$ element of $Q_{i}$.

(d) $\vec{\Phi}=\left\{\Phi_{j, i}\right\}_{1 \leq j \leq n ; 0 \leq i \leq m-1}$.

The following equation is an example for the case $n=3, m=3$ :

\section{Example 3.2.}

$$
\left(\begin{array}{l}
U_{1,0}^{\prime}(t) \\
U_{2,0}^{\prime}(t) \\
U_{3,0}^{\prime}(t) \\
U_{1,1}^{\prime}(t) \\
U_{2,1}^{\prime}(t) \\
U_{3,1}^{\prime}(t) \\
U_{1,2}^{\prime}(t) \\
U_{2,2}^{\prime}(t) \\
U_{3,2}^{\prime}(t)
\end{array}\right)=\left(\begin{array}{cccc} 
& & & \\
P_{0} & 0 & 0 \\
& & \\
Q_{1} & P_{1} & 0 \\
& & & \\
0 & & Q_{2} & P_{2}
\end{array}\right) \cdot\left(\begin{array}{l}
U_{1,0}(t) \\
U_{2,0}(t) \\
U_{3,0}(t) \\
U_{1,1}(t) \\
U_{2,1}(t) \\
U_{3,1}(t) \\
U_{1,2}(t) \\
U_{2,2}(t) \\
U_{3,2}(t)
\end{array}\right)
$$


where

$$
\begin{array}{r}
P_{0}=\left(\begin{array}{ccc}
-\mu_{1,0} & 2 p_{1,2,0} \beta_{2,0} & 2 p_{1,3,0} \beta_{3,0} \\
0 & -\mu_{2,0} & 2 p_{2,3,0} \beta_{3,0} \\
0 & 0 & -\mu_{3,0}
\end{array}\right) \\
P_{1}=\left(\begin{array}{ccc}
-\mu_{1,1} & 2 p_{1,2,1} \beta_{2,1} & 2 p_{1,3,1} \beta_{3,1} \\
0 & -\mu_{2,1} & 2 p_{2,3,1} \beta_{3,1} \\
0 & 0 & -\mu_{3,1}
\end{array}\right) \\
P_{2}=\left(\begin{array}{ccc}
-\mu_{1,2} & 2 p_{1,2,2} \beta_{2,2} & 2 p_{1,3,2} \beta_{3,2} \\
0 & -\mu_{2,2} & 2 p_{2,3,2} \beta_{3,2} \\
0 & 0 & -\mu_{3,2}
\end{array}\right) \\
Q_{1}=\left(\begin{array}{ccc}
2 q_{1,1,0} \beta_{1,0} & 2 q_{1,2,0} \beta_{2,0} & 2 q_{1,3,0} \beta_{3,0} \\
0 & 2 q_{2,2,0} \beta_{2,0} & 2 q_{2,3,0} \beta_{3,0} \\
0 & 0 & 2 q_{3,3,0} \beta_{3,0}
\end{array}\right) \\
Q_{2}=\left(\begin{array}{ccc}
2 q_{1,1,1} \beta_{1,1} & 2 q_{1,2,1} \beta_{2,1} & 2 q_{1,3,1} \beta_{3,1} \\
0 & 2 q_{2,2,1} \beta_{2,1} & 2 q_{2,3,1} \beta_{3,1} \\
0 & 0 & 2 q_{3,3,1} \beta_{3,1}
\end{array}\right)
\end{array}
$$

So, $A=$

$$
\left(\begin{array}{c}
-\mu_{1,0} \\
0 \\
0 \\
2 q_{1,1,0} \beta_{1,0} \\
0 \\
0 \\
0 \\
0 \\
0
\end{array}\right.
$$
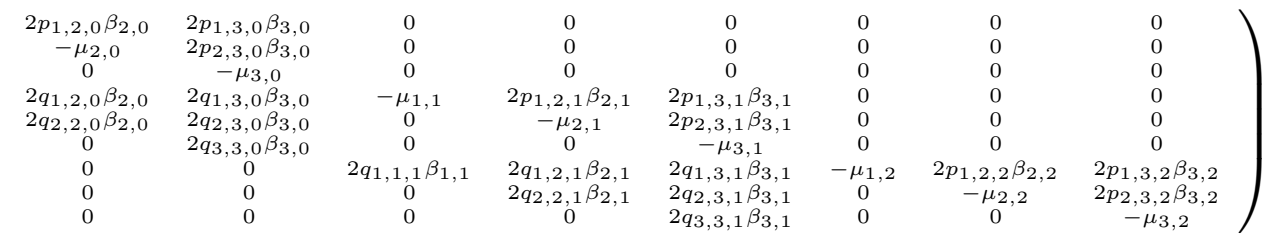

In whole generality, the solution to equation 3.4 with initial condition 3.5 is:

$$
\vec{U}(t)=e^{t A} \vec{\Phi}
$$

Theorem 3.3. Let hypotheses 2.1 and 3.1 hold. Then:

1. If, for every $1 \leq j \leq n$ and for every $0 \leq i \leq m-1, \mu_{j, i}>0$, then $\lim _{t \rightarrow \infty} U_{j, i}(t)=0$.

2. If, for every $1 \leq j \leq n$ and for every $0 \leq i \leq m-1, \mu_{j, i}=0$, then $U_{j, i}(t)$ is a polynomial in $t$ of degree $n-j+i$. Furthermore, the coefficient of $t^{n-j+i}$ of this polynomial is a multiple of $\Phi_{n, 0}$.

Proof. Case 1: $\forall 1 \leq j \leq n, 0 \leq i \leq m-1, \mu_{j, i}>0$

Matrix $A$ is a lower-triangular block matrix. Therefore, from [8], $\operatorname{det}\left(A-\lambda I_{N}\right)=\prod_{i=0}^{m-1} \operatorname{det}\left(P_{i}-\right.$ $\left.\lambda I_{n}\right)$, where $P_{i}$ are the matrices described above and $I_{k}$ is the identity $k \times k$ matrix $(k=n, N)$. Each $P_{i}$ is an upper-triangular matrix. Therefore, $\operatorname{det}\left(P_{i}-\lambda I_{n}\right)=\Pi_{j=1}^{n}\left(-\mu_{j, i}-\lambda\right)$. So, $\operatorname{det}\left(A-\lambda I_{N}\right)=$ $\Pi_{i=0}^{m-1} \Pi_{j=1}^{n}\left(-\mu_{j, i}-\lambda\right)$. The equation implies that eigenvalues of matrix $A$ are $\left\{-\mu_{j, i}\right\}_{1 \leq j \leq n ; 0 \leq i \leq m-1}$. Since they are all negative, $\lim _{t \rightarrow \infty} e^{t A} \vec{\Phi}=0$ (see e.g. [34]). Hence, $\lim _{t \rightarrow \infty} U_{j, i}(t)=0$.

Case 2: $\forall 1 \leq j \leq n, 0 \leq i \leq m-1, \mu_{j, i}=0$

Note, for the remainder of the proof: 
1. A block refers to an $n \times n$ matrix within the block matrix $A$ and the powers of $A$. $A$ is an $m \times m$ block matrix and so are its powers.

2. Consider an indexing on the block subdiagonals of a matrix such that the main block diagonal is the $0^{\text {th }}$ subdiagonal.

3. Consider an indexing on the superdiagonals of the blocks such that the main diagonal is the $0^{\text {th }}$ superdiagonal.

4. $M_{k, l}$ refers to the $(k, l)^{t h}$ block of block-matrix $M$, where $\mathrm{k}$ is the block row, $1 \leq k \leq m$ and 1 is the block-column $1 \leq l \leq m$.

5. $P_{i}$ and $Q_{i+1}, 0 \leq i \leq m-1$ are the same matrices defined earlier in the section but with one important difference: $P_{i}$ 's main diagonal is zero.

6. All non-zero elements of the matrices we consider are positive.

7. For the remainder of the proof, a matrix is considered "positive", if it is non-zero and all of its non-zero elements are positive.

8. A block row, a block column, or a block subdiagonal is referred to as "positive" if it consists of matrices that are "positive", according to the definition above.

Throughout the proof, we use a Claim, which is easy to prove and will be demonstrated by example in the Appendix:

Claim: Let $C$ and $B$ be $n \times n$ non-zero upper-triangular matrices. Let the first $k$ superdiagonals of $C$ (the main diagonal is also considered a superdiagonal) be zero and the first $m$ superdiagonals of $B$ be zero, $0 \leq k, m \leq n-1$. Assume every element on the $l^{\text {th }}$ superdiagonal of $C$, adopting the indexing from above, $l \geq k$, and $r^{t h}$ superdiagonal of $B, r \geq m$ is positive. Then $C B$ and $B C$ are upper-triangular matrices in which exactly the first $k+m$ superdiagonals are zero (if $k+m \geq n$, $C B$ and $B C$ will be the zero matrix) and every element of the $s^{t h}$ superdiagonal, $s \geq k+m$ is positive.

We encourage the reader to refer to Example 3.4 as an illustration of the Lemmas that follow.

Example 3.4. $(n=3, m=3)$ :

$$
\begin{aligned}
& P_{0}=\left(\begin{array}{ccc}
0 & 2 p_{1,2,0} \beta_{2,0} & 2 p_{1,3,0} \beta_{3,0} \\
0 & 0 & 2 p_{2,3,0} \beta_{3,0} \\
0 & 0 & 0
\end{array}\right) \\
& P_{1}=\left(\begin{array}{ccc}
0 & 2 p_{1,2,1} \beta_{2,1} & 2 p_{1,3,1} \beta_{3,1} \\
0 & 0 & 2 p_{2,3,1} \beta_{3,1} \\
0 & 0 & 0
\end{array}\right) \\
& P_{2}=\left(\begin{array}{ccc}
0 & 2 p_{1,2,2} \beta_{2,2} & 2 p_{1,3,2} \beta_{3,2} \\
0 & 0 & 2 p_{2,3,2} \beta_{3,2} \\
0 & 0 & 0
\end{array}\right)
\end{aligned}
$$




$$
\begin{aligned}
Q_{1} & =\left(\begin{array}{ccc}
2 q_{1,1,0} \beta_{1,0} & 2 q_{1,2,0} \beta_{2,0} & 2 q_{1,3,0} \beta_{3,0} \\
0 & 2 q_{2,2,0} \beta_{2,0} & 2 q_{2,3,0} \beta_{3,0} \\
0 & 0 & 2 q_{3,3,0} \beta_{3,0}
\end{array}\right) \\
Q_{2} & =\left(\begin{array}{ccc}
2 q_{1,1,1} \beta_{1,1} & 2 q_{1,2,1} \beta_{2,1} & 2 q_{1,3,1} \beta_{3,1} \\
0 & 2 q_{2,2,1} \beta_{2,1} & 2 q_{2,3,1} \beta_{3,1} \\
0 & 0 & 2 q_{3,3,1} \beta_{3,1}
\end{array}\right)
\end{aligned}
$$

$A=$

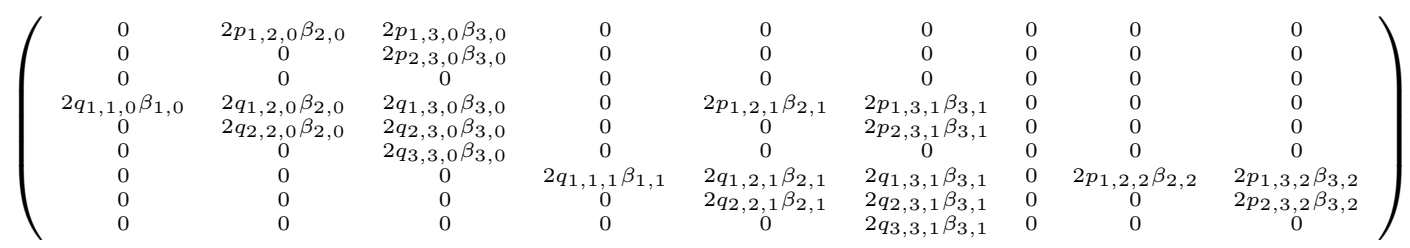

$$
A=\left(\begin{array}{ccc}
P_{0} & 0 & 0 \\
Q_{1} & P_{1} & 0 \\
0 & Q_{2} & P_{2}
\end{array}\right)
$$

$$
A^{2}=\left(\begin{array}{ccc}
P_{0}^{2} & 0 & 0 \\
Q_{1} P_{0}+P_{1} Q_{1} & P_{1}^{2} & 0 \\
Q_{2} Q_{1} & Q_{2} P_{1}+P_{2} Q_{2} & P_{2}^{2}
\end{array}\right)
$$

$$
P_{0}^{2}=\left(\begin{array}{ccc}
0 & 0 & 4 \beta_{2,0} \beta_{3,0} p_{1,2,0} p_{2,3,0} \\
0 & 0 & 0 \\
0 & 0 & 0
\end{array}\right)
$$

$$
\begin{gathered}
Q_{1} P_{0}+P_{1} Q_{1}= \\
\left(\begin{array}{ccc}
0 & 4 \beta_{1,0} \beta_{2,0} p_{1,2,0} q_{1,1,0}+4 \beta_{2,0} \beta_{2,1} p_{1,2,1} q_{2,2,0} & 4 \beta_{1,0} \beta_{3,0} p_{1,3,0} q_{1,1,0}+\underset{1}{4 \beta_{2,0} \beta_{3,0} p_{2,3,0} q_{1,2,0}+4 \beta_{2,1} \beta_{3,0} p_{1,2,1} q_{2,3,0}}+4 \beta_{3,0} \beta_{3,1} p_{1,3,1} q_{3,3,0} \\
0 & 0 & 4 \beta_{2,0} \beta_{3,0} p_{2,3,0} q_{2,2,0}+4 \beta_{3,0} \beta_{3,1} p_{2,3,1} q_{3,3,0} \\
0 & 0 & 0
\end{array}\right)
\end{gathered}
$$

$$
Q_{2} Q_{1}=
$$

$\left(\begin{array}{ccc}4 \beta_{1,0} \beta_{1,1} q_{1,1,0} q_{1,1,1} & 4 \beta_{1,1} \beta_{2,0} q_{1,1,1} q_{1,2,0}+4 \beta_{2,0} \beta_{2,1} q_{1,2,1} q_{2,2,0} & 4 \beta_{1,1} \beta_{3,0} q_{1,1,1} q_{1,3,0}+4 \beta_{2,1} \beta_{3,0} q_{1,2,1} q_{2,3,0}+4 \beta_{3,0} \beta_{3,1} q_{1,3,1} q_{3,3,0} \\ 0 & 4 \beta_{2,0} \beta_{2,1} q_{2,2,0} q_{2,2,1} & 4 \beta_{2,1} \beta_{3,0} q_{2,2,1} q_{2,3,0}+4 \beta_{3,0} \beta_{3,1} q_{2,3,1} q_{3,3,0} \\ 0 & 0 & 4 \beta_{3,0} \beta_{3,1} q_{3,3,0} q_{3,3,1}\end{array}\right)$

$$
A^{3}=\left(\begin{array}{ccc}
0 & 0 & 0 \\
Q_{1} P_{0}^{2}+P_{1} Q_{1} P_{0}+P_{1}^{2} Q_{1} & 0 & 0 \\
Q_{2} Q_{1} P_{0}+Q_{2} P_{1} Q_{1}+P_{2} Q_{2} Q_{1} & Q_{2} P_{1}^{2}+P_{2}^{2} Q_{2}+P_{2} Q_{2} P_{1} & 0
\end{array}\right)
$$

$$
P_{0}^{3}=\left(\begin{array}{lll}
0 & 0 & 0 \\
0 & 0 & 0 \\
0 & 0 & 0
\end{array}\right)
$$

$Q_{1} P_{0}^{2}+P_{1} Q_{1} P_{0}+P_{1}^{2} Q_{1}=$

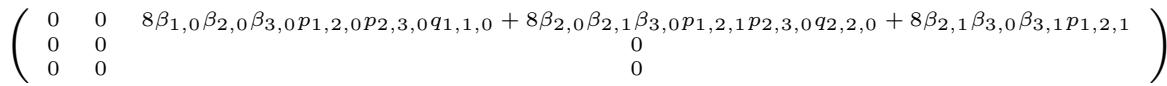




$$
\begin{aligned}
Q_{2} Q_{1} P_{0}+Q_{2} P_{1} Q_{1}+P_{2} Q_{2} Q_{1}= & \left(\begin{array}{ccc}
0 & c_{1,2} & c_{1,3} \\
0 & 0 & c_{2,3} \\
0 & 0 & 0
\end{array}\right),
\end{aligned}
$$

where

$$
\begin{aligned}
& c_{1,2}=8 \beta_{1,0} \beta_{1,1} \beta_{2,0} p_{1,2,0} q_{1,1,0} q_{1,1,1}+8 \beta_{1,1} \beta_{2,0} \beta_{2,1} p_{1,2,1} q_{1,1,1} q_{2,2,0}+8 \beta_{2,0} \beta_{2,1} \beta_{2,2} p_{1,2,2} q_{2,2,0} q_{2,2,1} \\
& c_{1,3}=8 \beta_{1,0} \beta_{1,1} \beta_{3,0} p_{1,3,0} q_{1,1,0} q_{1,1,1}+2 \beta_{3,0} p_{2,3,0}\left(4 \beta_{1,1} \beta_{2,0} q_{1,1,1} q_{1,2,0}+4 \beta_{2,0} \beta_{2,1} q_{1,2,1} q_{2,2,0}\right)+ \\
& +8 \beta_{1,1} \beta_{2,1} \beta_{3,0} p_{1,2,1} q_{1,1,1} q_{2,3,0}+8 \beta_{2,1} \beta_{2,2} \beta_{3,0} p_{1,2,2} q_{2,2,1} q_{2,3,0}+ \\
& +2 \beta_{3,0}\left(4 \beta_{1,1} \beta_{3,1} p_{1,3,1} q_{1,1,1}+4 \beta_{2,1} \beta_{3,1} p_{2,3,1} q_{1,2,1}\right) q_{3,3,0}+ \\
& +2 \beta_{3,0} q_{3,3,0}\left(4 \beta_{2,2} \beta_{3,1} p_{1,2,2} q_{2,3,1}+4 \beta_{3,1} \beta_{3,2} p_{1,3,2} q_{3,3,1}\right) \\
& c_{2,3}=8 \beta_{2,0} \beta_{2,1} \beta_{3,0} p_{2,3,0} q_{2,2,0} q_{2,2,1}+8 \beta_{2,1} \beta_{3,0} \beta_{3,1} p_{2,3,1} q_{2,2,1} q_{3,3,0}+8 \beta_{3,0} \beta_{3,1} \beta_{3,2} p_{2,3,2} q_{3,3,0} q_{3,3,1}
\end{aligned}
$$

$$
\begin{aligned}
& A^{4}= \\
& \left(\begin{array}{crc}
0 & 0 & 0 \\
0 & 0 & 0 \\
Q_{2} Q_{1} P_{0}^{2}+Q_{2} P_{1} Q_{1} P_{0}+P_{2} Q_{2} Q_{1} P_{0}+Q_{2} P_{1}^{2} Q_{1}+P_{2}^{2} Q_{2} Q_{1}+P_{2} Q_{2} P_{1} Q_{1} & 0 & 0
\end{array}\right) \\
& Q_{2} Q_{1} P_{0}^{2}+Q_{2} P_{1} Q_{1} P_{0}+P_{2} Q_{2} Q_{1} P_{0}+Q_{2} P_{1}^{2} Q_{1}+P_{2}^{2} Q_{2} Q_{1}+P_{2} Q_{2} P_{1} Q_{1}= \\
& \left(\begin{array}{ccc}
0 & 0 & c_{1,3}^{1} \\
0 & 0 & 0 \\
0 & 0 & 0
\end{array}\right)
\end{aligned}
$$

where

$c_{1,3}^{1}=16 \beta_{1,0} \beta_{1,1} \beta_{2,0} \beta_{3,0} p_{1,2,0} p_{2,3,0} q_{1,1,0} q_{1,1,1}+16 \beta_{1,1} \beta_{2,0} \beta_{2,1} \beta_{3,0} p_{1,2,1} p_{2,3,0} q_{1,1,1} q_{2,2,0}+$

$+16 \beta_{2,0} \beta_{2,1} \beta_{2,2} \beta_{3,0} p_{1,2,2} p_{2,3,0} q_{2,2,0} q_{2,2,1}+16 \beta_{1,1} \beta_{2,1} \beta_{3,0} \beta_{3,1} p_{1,2,1} p_{2,3,1} q_{1,1,1} q_{3,3,0}+$

$+16 \beta_{2,1} \beta_{2,2} \beta_{3,0} \beta_{3,1} p_{1,2,2} p_{2,3,1} q_{2,2,1} q_{3,3,0}+16 \beta_{2,2} \beta_{3,0} \beta_{3,1} \beta_{3,2} p_{1,2,2} p_{2,3,2} q_{3,3,0} q_{3,3,1}$

$$
A^{5}=\left(\begin{array}{lll}
0 & 0 & 0 \\
0 & 0 & 0 \\
0 & 0 & 0
\end{array}\right)
$$

The proof also uses the following three lemmas, which are proved in the Appendix:

Lemma 3.5. If $1 \leq k \leq m-1$, the $k^{\text {th }}$ block subdiagonal is the highest indexed positive block subdiagonal of $A^{k}$. Any block subdiagonal of higher index is 0 .

Lemma 3.6. In $A^{k}, 1 \leq k \leq m+n$, any block element of a positive block subdiagonal indexed by $i, 0 \leq i \leq m-1$, is an upper-triangular matrix in which exactly the first $k-i$ superdiagonals (including the main diagonal) are 0.

Lemma 3.7. If $n \leq k<n+m$, the $(k-n+1)^{\text {th }}$ block subdiagonal is the lowest indexed positive block subdiagonal of $A^{k}$. Any block subdiagonal of lower index is 0. 
All eigenvalues of $A$ are zero, therefore, $A^{n+m}=0, A$ is nilpotent, and $e^{t A} \vec{\Phi}$ is a finite polynomial vector in $t$. Thus, each cell class has a polynomial growth in $t$.

First, we need to determine the polynomials in $t$ that describe the growth of each mutation class. This is equivalent to determining the polynomials in $t$ that corresponds to a block-row $i$ in $e^{t A}=I+t A+\frac{t^{2} A^{2}}{2}+\ldots+\frac{t^{m+n-1} A^{m+n-1}}{(m+n-1) !}, 1 \leq i \leq m$. This is, in turn, equivalent to determining for which powers $k$ of $A^{k}$, the block row corresponding to the mutation class is positive.

From Lemma 3.5, the power $k$, for which a block subdiagonal indexed $(i-1)$ in $A^{k}$ becomes positive (i.e. the power for which this block becomes the highest indexed positive block in $A^{k}$ ) is $(i-1)$. So, any polynomial in $t$ that describes the growth of cells in mutation class corresponding to block row $i, 1 \leq i \leq m$, is at least $t^{i-1}$.

From Lemma 3.7, it follows that a block row $i, 1 \leq i \leq m$, becomes 0 only when the $(i-1)^{t h}$ block subdiagonal,which contains the block element $A_{i, 1}^{k}$, becomes 0 for some power $k$ of $A^{k}, k \geq n$. Therefore, one can conclude that when $k=n+i-2$, the $(i-1)^{t h}$ block subdiagonal is positive in $A^{n+i-2}$ and when $k=n+i-1$, it is 0 in $A^{n+i-1}$. Therefore, any polynomial in $t$ that describes the growth of cells in mutation class corresponding to block row $i, 1 \leq i \leq m$, is at most $t^{n+i-2}$.

What needs to be determined next is what polynomials describe the growth of the cells within the telomere classes of mutation class $i-1$. This is equivalent to determining the highest power of $\mathrm{k}$ for which a row $j, 1 \leq j \leq n$ remains positive in $A_{i, 1}^{k}, i-1 \leq k \leq n+i-1$. The last statement, in turn, is equivalent to determining the highest power of $k$ for which the superdiagonal indexed $n-j$ remains positive in $A_{i, 1}^{k}$. From Lemma 3.6, this power is $k=i+n-j-1$. Therefore, the row $j$ in block row $i$ corresponds to a polynomial in $t$ of power $t^{i+n-j-1}, 1 \leq i \leq m, 1 \leq j \leq n$. If we switch the indexing of $i$ to make it correspondent to how many mutations a class of cells a block row represents, the growth of cells in mutation class $i, 0 \leq i \leq m-1$, telomere class $j, 1 \leq j \leq n$ is determined by a polynomial in $t$ of power $t^{i+n-j}$.

Example 3.8. $(n=2, m=2)$ :

$$
\begin{gathered}
A=\left(\begin{array}{cccc}
0 & 2 p_{1,2,0} \beta_{2,0} & 0 & 0 \\
0 & 0 & 0 & 0 \\
2 q_{1,1,0} \beta_{1,0} & 2 q_{1,2,0} \beta_{2,0} & 0 & 2 p_{1,2,1} \beta_{2,1} \\
0 & 2 q_{2,2,0} \beta_{2,0} & 0 & 0
\end{array}\right) \\
\left(\begin{array}{c}
U_{1,0} \\
U_{2,0} \\
U_{1,1} \\
U_{2,1}
\end{array}\right)=e^{t A} \cdot \vec{\Phi}= \\
\Phi_{1,0}+2 p_{2,1,0} \beta_{2,0} \Phi_{2,0} t \\
\Phi_{2,0} \\
=\left(\begin{array}{c}
\Phi_{1,1}+2 p_{2,1,1} \beta_{2,1} \Phi_{2,1} t+2 \Phi_{1,0} q_{1,1,0} \beta_{1,0} t+2 \Phi_{2,0}\left(q_{1,2,0} \beta_{2,0} t+p_{2,1,0} \beta_{2,0} q_{1,1,0} \beta_{1,0} t^{2}+p_{2,1,1} \beta_{2,1} q_{2,2,0} \beta_{2,0} t^{2}\right) \\
\Phi_{2,1}+2 \Phi_{2,0} q_{2,2,0} \beta_{2,0} t
\end{array}\right)
\end{gathered}
$$

From Lemma 3.6 and the above, the element that contains the highest power of $t, t^{i+n-j}$, in row $j$, block row $i$ in $e^{t A}, 1 \leq j \leq n ; 1 \leq i \leq m$, is in the $n^{t h}$ column of block $e_{i, 1}^{t A}$. This column contains the elements that are matrix multiplied by $\Phi_{n, 0}$ when determining $e^{t A} \vec{\Phi}$. Hence, the coefficient of $t^{n-j+i}, 1 \leq j \leq n ; 0 \leq i \leq m-1$, is a multiple of $\Phi_{n, 0}$ 
In Theorem 3.3.1, the model depicts a situation in which cell mortality has profound effect on the outcome - eventually all cells die. It can predict the cell dynamics in a long-term in vitro or in vivo experiment. Theorem 3.3.2 presents a model, more suitable to depict short term in vitro and in vivo interaction between the different cell classes, because cell mortality does not play a major role in short term experiments. Part 2 of the theorem shows that the more differentiated a cell population is, the faster it grows. This result is consistent with our knowledge of the distribution of cells within the cell hierarchy of the human body, for example the hierarchy of human hematopoiesis [30]. Further, the theorem also shows that cell populations that have acquired a higher number of malignant mutations grow faster. This result is consistent with the nature of aggressive cancer growth [49][39][25]. Note that Theorem 3.3 makes no assumption about the proliferation rate, $\beta_{j, i}$, of different classes. Therefore, the increased growth can be explained by the processes of differentiation and mutation accumulation. In the model, those processes are presented in Equation 2.2, which describes the sources of new cells for the different cell classes. This fact suggests that mutation acquisition is reason enough for cancer cells to grow faster than normal cells. A more complete treatment of the issue is given in the Discussion section.

\section{Nonlinear Case}

We introduce a crowding term in equation 3.4:

$$
\begin{gathered}
\vec{U}^{\prime}(t)=A \vec{U}(t)-F(\vec{U}(t)) \vec{U}(t) \\
\vec{U}(0)=\vec{\Phi},
\end{gathered}
$$

where $A$ is the matrix defined in the linear case, $\vec{U}(t)$, as before, is the vector representing the total number of cells of the different telomere and mutation classes, $\vec{\Phi}$ is the vector of the initial cell populations, and for fixed $t \geq 0, F$ is a positive linear functional from $L_{1}\left(\mathbb{R}_{+}^{N}\right)$ to $\mathbb{R}_{+}$. The "crowding" term, $F(\vec{U}(t)) \vec{U}(t)$, describes a situation under which the growth of the total cell population, or the population of certain cell classes, slows down. The biological interpretations are numerous. Crowding can occur because of limited nutrients, space, or events during which cells get signals to cease proliferation. The model presented in Equation 4.1 does not choose a specific crowding term in this section. In the next section, Numerical Simulations, we choose a specific linear functional $F$ and a biological interpretation. However, the mathematical results that follow do not depend on the biological interpretation of the crowding term. The following assumptions hold:

Hypothesis 4.1.

1. $\mu_{j, i}(a)=\mu_{j, i}>0, \forall 1 \leq j \leq n ; 0 \leq i \leq m-1$

2. $\beta_{j, i}(a)=\beta_{j, i}>0, \forall 1 \leq j \leq n ; 0 \leq i \leq m-1$

3. $p_{j, k, i}=0$ for $j>k, \forall 1 \leq j \leq n ; 0 \leq i \leq m-1$. 
Note: $p_{j, j, i}$ need not equal $\frac{1}{2}, \forall 1 \leq j \leq n, 0 \leq i \leq m-1$

By an argument similar to the one in the proof of Theorem 3.3, Case 1, the eigenvalues of $A$ are of the form: $-\mu_{j, i}-\beta_{j, i}+2 p_{j, j, i} \beta_{j, i}$. Let $\lambda_{0}$ denote the dominant eigenvalue, that is $\lambda_{0}>\lambda$, where $\lambda$ is any other eigenvalue of $A$.

Hypothesis 4.2. $\lambda_{0}=-\mu_{n, m-1}-\beta_{n, m-1}+2 p_{n, n, m-1} \beta_{n, m-1}$.

In other words, the dominant eigenvalue is associated with the growth of the cancer stem cells. We can assume this since stem cells have higher degree of self-renewal than other cells, hence $p_{n, n, m-1} \geq p_{j, j, i}, \forall 1 \leq j \leq n ; 0 \leq i \leq m-1$ [44][24][43]. Also, cancer cells are assumed to have higher proliferation rate than normal cells, hence $\beta_{n, m-1} \geq \beta_{j, i}, \forall 1 \leq j \leq n, 0 \leq i \leq m-1$ [12][37][44][24]. Note that if $p_{n, n, m-1}>\frac{1}{2}$ and $\beta_{n, m-1}>\frac{\mu_{n, m-1}}{2 p_{n, n, m-1}-1}$, then $\lambda_{0}>0$.

Let $\lambda_{0}>0$. Let $A^{\prime} \stackrel{\text { def }}{=} A-\lambda_{0} I$. $A^{\prime}$ is a block matrix with structure similar to $A$, the only difference being the diagonal entries, hence the only difference being the $P_{i}$ blocks. For simplicity of notation, let:

$$
\bar{m}_{j, i} \stackrel{\text { def }}{=}-\mu_{j, i}-\beta_{j, i}+2 p_{j, j, i} \beta_{j, i}-\left(-\mu_{n, m-1}-\beta_{n, m-1}+2 p_{n, n, m-1} \beta_{n, m-1}\right)
$$

and

$$
\bar{p}_{j, k, i} \stackrel{\text { def }}{=} 2 p_{j, k, i} \beta_{k, i}, 1 \leq j \leq n-1,0 \leq i \leq m-1, k>j .
$$

Then, for $0 \leq i<m-1$,

$$
P_{i}^{\prime} \stackrel{\text { def }}{=}\left(\begin{array}{cccc}
\bar{m}_{1, i} & \bar{p}_{1,2, i} & \ldots & \ldots \\
0 & \bar{m}_{2, i} & \bar{p}_{2,3, i} & \ddots \\
\vdots & \ddots & \ddots & \ddots \\
0 & \ldots & \ldots & \bar{m}_{n, i}
\end{array}\right)
$$

For $i=m$,

$$
P_{m-1}^{\prime} \stackrel{\text { def }}{=}\left(\begin{array}{cccc}
\bar{m}_{1, i} & \bar{p}_{1,2, i} & \ldots & \ldots \\
0 & \bar{m}_{2, i} & \bar{p}_{2,3, i} & \ddots \\
\vdots & \ddots & \ddots & \ddots \\
0 & \ldots & \ldots & 0
\end{array}\right)
$$

Therefore,

$$
A^{\prime}=\left(\begin{array}{cccc}
P_{0}^{\prime} & 0 & \ldots & 0 \\
Q_{1} & P_{1}^{\prime} & \ldots & \vdots \\
\ddots & \ddots & \ddots & \ddots \\
0 & \ldots & Q_{n} & P_{m-1}^{\prime}
\end{array}\right)
$$

Note: In the absence of any differentiability assumption on $F$, it is not possible to apply the Fundamental Theorem on Existence and Uniqueness of Solutions. Therefore, the following theorem uses previous results in order to show the existence and uniqueness of the solution of Equation 4.1 . 
Theorem 4.3. There is a unique solution to Equation 4.1 and the eigenspace of the dominant eigenvalue $\lambda_{0}$ of $A$ is one dimensional. Further, the first $N-n$ entries of $\vec{\Psi}$ are 0 , the last $n$ are non-zero, and $\lim _{t \rightarrow \infty} \vec{U}(t)=\frac{\lambda_{0} \Pi_{0} \vec{\Phi}}{F\left(\Pi_{0} \vec{\Phi}\right)}=\frac{\lambda_{0} \vec{\Psi}}{F(\vec{\Psi})}$, where $\Pi_{0}$ is the eigenprojection associated with $\lambda_{0}$, $\vec{U}(t)$ is the unique solution to Equation 4.1, and $\vec{\Psi}$ is an eigenvector of $\lambda_{0}$.

Proof. By [47],[19], and [18], Equation 4.1 has a unique solution, $\vec{U}(t)$, and since $\lambda_{0}>0$, $\lim _{t \rightarrow \infty} \vec{U}(t)=$ $\frac{\lambda_{0} \Pi_{0} \vec{\Phi}}{F\left(\Pi_{0} \vec{\Phi}\right)}$.

We are interested in finding the eigenvector of $\lambda_{0}$, in other words $\vec{\Psi} \in \mathbb{R}^{N}$, such that $A^{\prime} \vec{\Psi}=0$. Let us initially restrict our attention to solutions of $P_{0}^{\prime} \vec{\Psi}_{0}=0$, where $\vec{\Psi}_{0}$ is the vector of the first $n$ entries of $\vec{\Psi}$. Refer to Example 4.4 throughout the proof.

Example 4.4. $(n=3)$ :

$$
\begin{gathered}
P_{0}^{\prime}=\left(\begin{array}{ccc}
\bar{m}_{1,0} & \bar{p}_{1,2,0} & \bar{p}_{1,3,0} \\
0 & \bar{m}_{2,0} & \bar{p}_{2,3,0} \\
0 & 0 & \bar{m}_{3,0}
\end{array}\right) \\
\vec{\Psi}_{0}=\left(\begin{array}{c}
\Psi_{1,0} \\
\Psi_{2,0} \\
\Psi_{3,0}
\end{array}\right)
\end{gathered}
$$

So,

$$
P_{0}^{\prime} \cdot \vec{\Psi}_{0}=\left(\begin{array}{c}
\bar{m}_{1,0} \Psi_{1,0}+\bar{p}_{1,2,0} \Psi_{2,0}+\bar{p}_{1,3,0} \Psi_{3,0} \\
\bar{m}_{2,0} \Psi_{2,0}+\bar{p}_{2,3,0} \Psi_{3,0} \\
\bar{m}_{3,0} \Psi_{3,0}
\end{array}\right)=0 .
$$

Hence, $\Psi_{3,0}=0 \Rightarrow \Psi_{2,0}=0 \Rightarrow \Psi_{1,0}=0$.

$$
\begin{gathered}
P_{m-1}^{\prime}=\left(\begin{array}{ccc}
\bar{m}_{1, m-1} & \bar{p}_{1,2, m-1} & \bar{p}_{1,3, m-1} \\
0 & \bar{m}_{2, m-1} & \bar{p}_{2,3, m-1} \\
0 & 0 & 0
\end{array}\right) \\
\vec{\Psi}_{m-1}=\left(\begin{array}{c}
\Psi_{1, m-1} \\
\Psi_{2, m-1} \\
\Psi_{3, m-1}
\end{array}\right) \\
P_{m-1}^{\prime} \cdot \vec{\Psi}_{m-1}=0
\end{gathered}
$$

hence $\Psi_{3, m-1}$ is a free variable, $\Psi_{2, m-1}=\frac{-\bar{p}_{2,3, m-1}}{\bar{m}_{2, m-1}} \Psi_{3, m-1}$,

and $\Psi_{1, m-1}=\left(\frac{\bar{p}_{1,2, m-1} \bar{p}_{2,3, m-1}}{\bar{m}_{1, m-1} \bar{m}_{2, m-1}}-\frac{\bar{p}_{1,3, m-1}}{\bar{m}_{1, m-1}}\right) \Psi_{3, m-1}$

$P_{0}^{\prime} \vec{\Psi}_{0}=0$ is a system of $n$ equations of $n$ variables. Note that since $P_{0}^{\prime}$ is positive and uppertriangular, each variable $\Psi_{j, 0}, 1 \leq j \leq n$ is linearly dependent on all variables $\Psi_{k, 0}, j<k \leq n$. The last equation of the system is $m_{n, 0} \vec{\Psi}_{n, 0}=0$, therefore $\vec{\Psi}_{n, 0}=0$. The $(n-1)^{t h}$ equation is 
$m_{n-1,0} \vec{\Psi}_{n-1,0}+\bar{p}_{n-1, n, 0} \vec{\Psi}_{n, 0}=0$, so $\vec{\Psi}_{n-1,0}=0$. By similar arguments for the other elements $\Psi_{j, 0}, 1 \leq j<n-1$, in $\vec{\Psi}_{0}, \vec{\Psi}_{0}=0$.

Let us consider the second block-row of $A^{\prime}$. We need to solve, in block-matrix form, the equation:

$$
\left(Q_{1} \mid P_{1}^{\prime}\right) \cdot\left(\begin{array}{c}
\Psi_{1,0} \\
\vdots \\
\Psi_{n, 1}
\end{array}\right)=0 .
$$

The first elements of $\left(\Psi_{1,0} \ldots \Psi_{n, 1}\right)^{T}=\overrightarrow{\Psi_{0}}$ are zero. So, from the argument above and the rules of matrix multiplication, $Q_{1}$ does not contribute to the solution of the system. Therefore, the equation above is reduced to :

$$
\left(P_{1}^{\prime}\right) \cdot\left(\begin{array}{c}
\Psi_{1,1} \\
\vdots \\
\Psi_{n, 1}
\end{array}\right)=0 .
$$

By an argument similar to the one above, $\left(\Psi_{1,1}, \ldots, \Psi_{n, 1}\right)^{T}=0$. By a similar inductive argument, it is easy to show that $\left(\Psi_{1,0} \ldots \Psi_{n, m-2}\right)^{T}=0$.

The solution to the equation $A^{\prime} \vec{\Psi}=0$ is reduced to solving:

$$
\left(P_{m-1}^{\prime}\right) \cdot\left(\begin{array}{c}
\Psi_{1, m-1} \\
\vdots \\
\Psi_{n, m-1}
\end{array}\right)=0
$$

which is a system of $n$ equations and $n$ variables, one of them free. Let, for convenience, the free variable be $\Psi_{n, m-1} . P_{m-1}^{\prime}$ is an upper-triangular, positive (except for its $n^{t h}$, last row, which is 0 ) matrix. Therefore, every variable $\Psi_{j, m-1}$ is linearly dependent on the variables $\Psi_{k, m-1}, j<k \leq n$. Therefore, each $\Psi_{l, m-1}, 1 \leq l \leq n$ can be expressed as a positive multiple of $\Psi_{n, m-1}$. So, the eigenvector associated with $\lambda_{0}, \overrightarrow{\vec{\Psi}}$, is unique up to a choice of $\Psi_{n, m-1}$. Its first $N-n$ entries are 0 and the last $n$ entries are positive multiples of $\Psi_{n, m-1}$. Fix $\Psi_{n, m-1}>0$ and let $\vec{\Psi}$ be the so defined eigenvector of the eigenspace of $A$, associated with $\lambda_{0}$. Thus, the eigenspace is one-dimensional. Hence $\frac{\lambda_{0} \Pi_{0} \vec{\Phi}}{F\left(\Pi_{0} \vec{\Phi}\right)}=\frac{\lambda_{0} C \vec{\Psi}}{F(C \vec{\Psi})}=\frac{\lambda_{0} C \vec{\Psi}}{C F(\vec{\Psi})}=\frac{\lambda_{0} \vec{\Psi}}{F(\vec{\Psi})}, C \in \mathbb{C} . F(\vec{\Psi})$ is a constant, hence $\lim _{t \rightarrow \infty} \vec{U}(t)=\frac{\lambda_{0} \vec{\Psi}}{F(\vec{\Psi})}$, an $\mathrm{N}$-dimensional vector, whose only non-zero elements correspond to the telomere classes of the cancer cells.

The biological interpretation of the result of Theorem 4.3 is that all cell populations converge to a steady state, in which only cancer cells survive. Given the assumptions about the higher proliferation rate and higher probability of self-renewal of the cancer stem cell, $p_{n, n, m}$, the cancer cells eventually overtake all other existing cells. However, they also reach an equilibrium determined by the fact that there is a crowding effect in the cell environment. Therefore, the cancer cells, by being more competitive, have taken over the somatic cellular tissue. However, because of limited resources they cannot increase their population indefinitely. 


\section{Numerical Simulation}

Table 1: Numerical values for parameters in the model

\begin{tabular}{l|c|r|}
\hline Parameter & $\begin{array}{c}\text { Range based } \\
\text { on references }\end{array}$ & $\begin{array}{r}\text { Simulation } \\
\text { Value }\end{array}$ \\
\hline $\begin{array}{l}\text { Non-cancer cell proliferation rate, } \beta_{j, i}, \\
1 \leq j \leq n, 0 \leq i \leq m-2 \text { (cells/week) [48][6] }\end{array}$ & $0.5-3.36$ & 0.65 (Stem Cell); \\
\hline Cancer cell proliferation rate, $\beta_{j, m-1}$ (cells/week) [39][49] & 0.35 to 13.44 & 0.7 (Non-Stem Cell) \\
\hline Cell mortality rate, $\mu_{j, i}$ (cells/week) [6][24] & $0.001-1.68$ & 0.001 (nonlinear simulation) \\
$1 \leq j \leq n, 0 \leq i \leq m-1$ (cells/week) & & 0 (linear simulation) \\
\hline $\begin{array}{l}\text { Mutation probability, } q_{j, k, i}[24] \\
1 \leq j \leq n, j \leq k \leq n, 0 \leq i \leq m-2\end{array}$ & 0.00001 & 0.00001 \\
\hline Stem Cell self-renewal probability, & & 0.65 (nonlinear simulation) \\
$p_{j, j, i}, j=n, 0 \leq i \leq m-1$ & & 0.5 (linear simulation) \\
\hline $\begin{array}{l}\text { Non-Stem Cell self-renewal probability, } \\
p_{j, j, i}, 1 \leq j \leq n-1,0 \leq i \leq m-1\end{array}$ & & 0.5 \\
\hline \hline
\end{tabular}

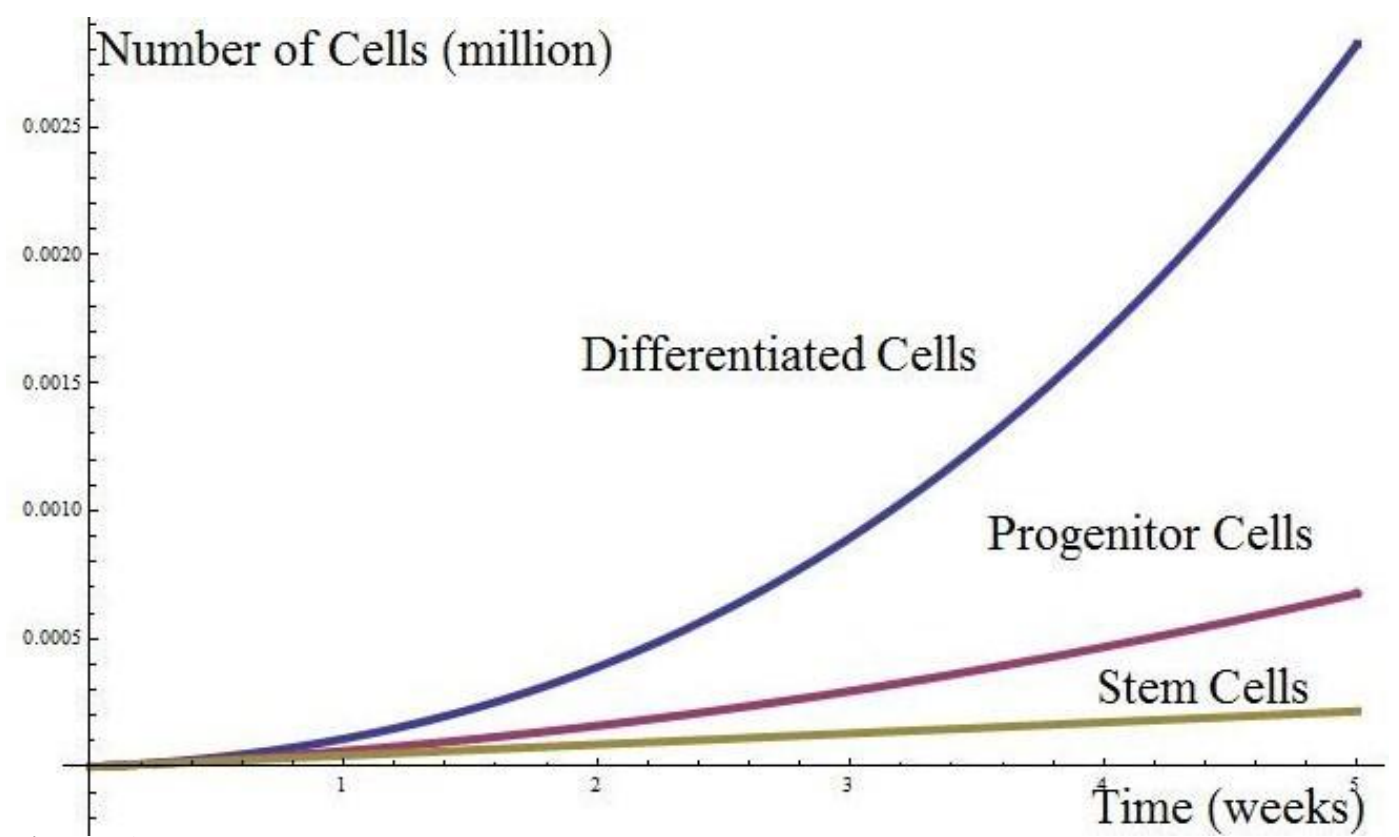

Figure 3: Linear model with $n=3$ maximum number of telomeres and $m=3$ mutation classes ( 2 mutations necessary to reach malignancy). Polynomial growth of cells with one mutation ( $i=1$ mutation), according to the results of Theorem 3.3.2. Stem cells $(j=3$ telomeres) grow linearly, progenitor cells $(j=2$ telomeres) in $t^{2}$, and differentiated cells $\left(j=1\right.$ telomere) in $t^{3}$.

The main purpose of this section is to show numerical simulations of the mathematical results and demonstrate them in a visual setting. In Table 1 one can see the parameters used, ranges for the values of the parameters, based on evidence or previous models, and the values used in the 


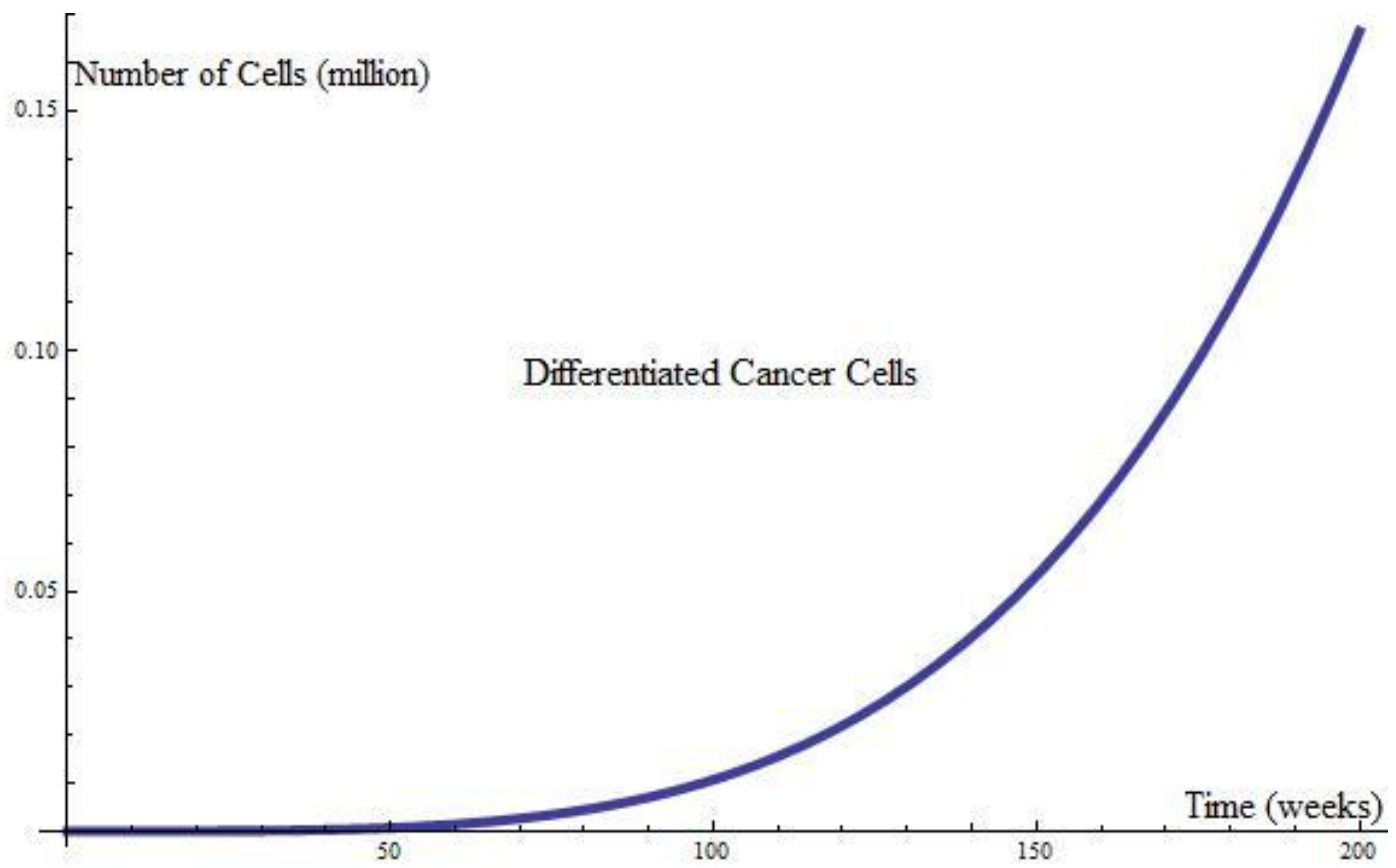

Figure 4: Linear model with $\mathrm{n}=3$ maximum number of telomeres and $\mathrm{m}=3$ mutation classes ( 2 mutations necessary to reach malignancy). Polynomial growth $\left(t^{4}\right)$ of differentiated cancer cells $(j=1$ telomere, $i=2$ mutations), according to the results of Theorem 3.3.2.

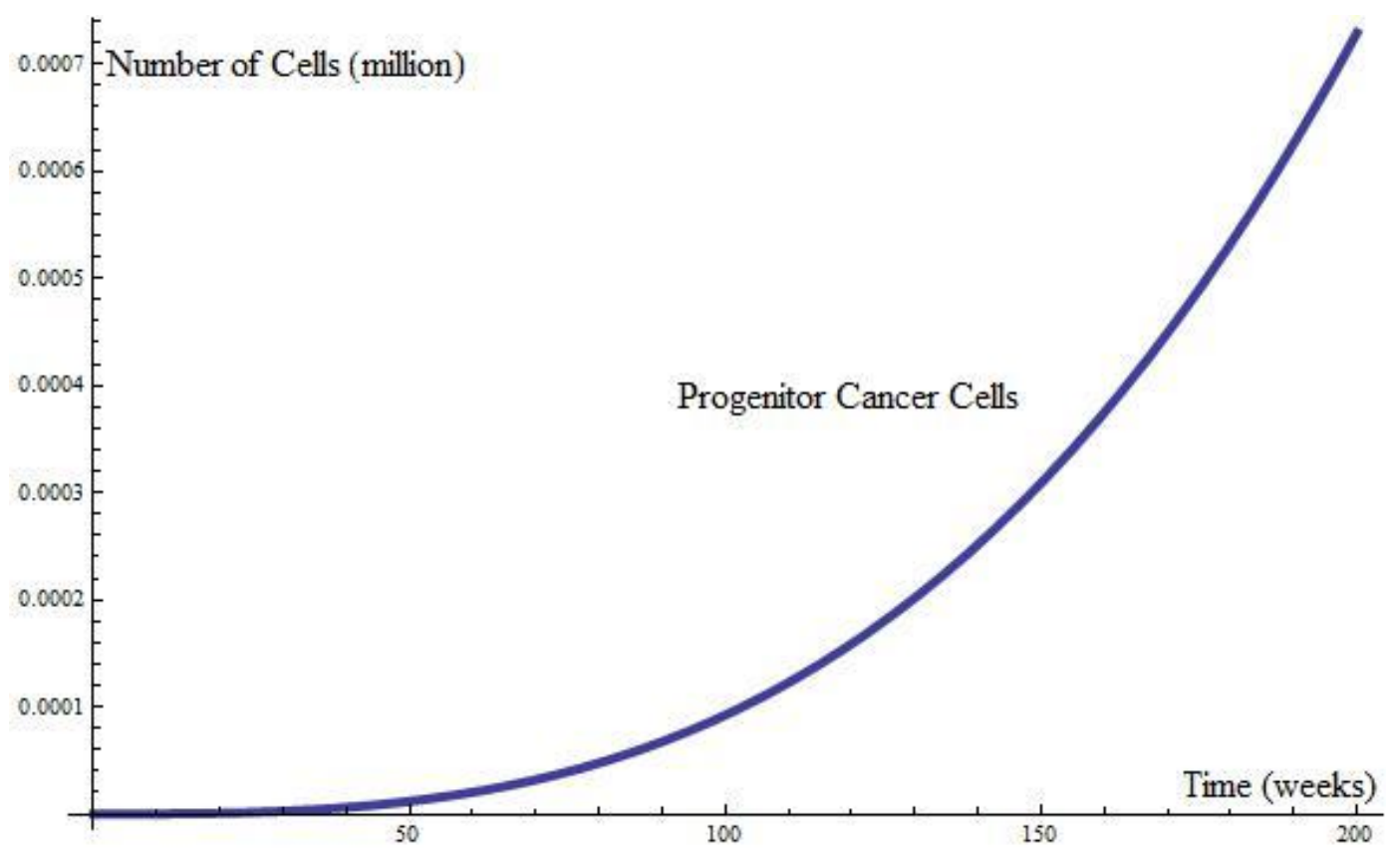

Figure 5: Linear model with $\mathrm{n}=3$ maximum number of telomeres and $\mathrm{m}=3$ mutation classes ( 2 mutations necessary to reach malignancy). Polynomial growth $\left(t^{3}\right)$ of progenitor cancer cells $(j=2$ telomeres, $i=2$ mutations), according to the results of Theorem 3.3.2. 


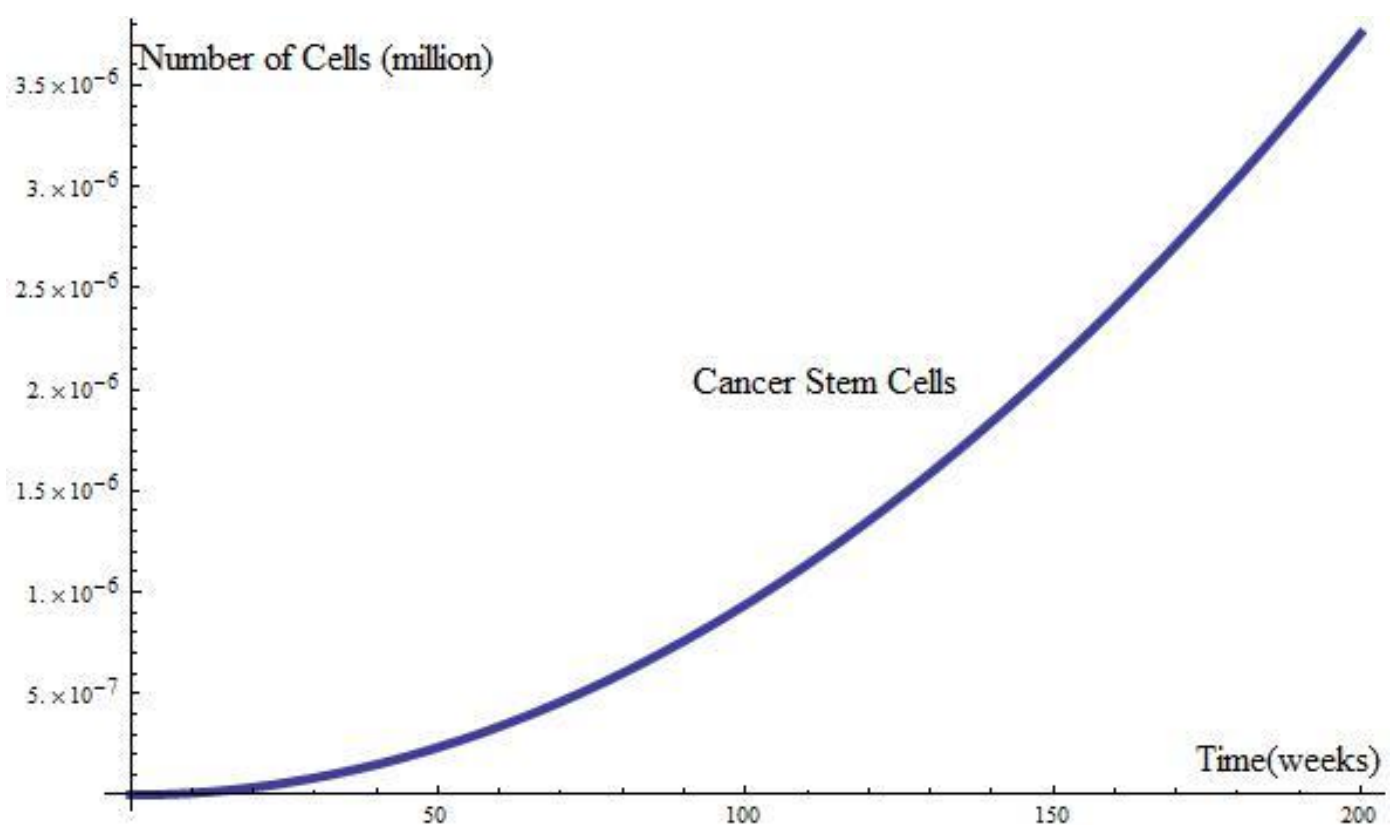

Figure 6: Linear model with $n=3$ maximum number of telomeres and $m=3$ mutation classes $(2$ mutations necessary to reach malignancy). Polynomial growth $\left(t^{2}\right)$ of cancer stem cells ( $j=3$ telomeres, $i=2$ mutations), according to the results of Theorem 3.3.2.

simulations.

The proliferation rate, $\beta_{j, i}$, depends on the type of cells it refers to (stem, differentiated, cancer, etc.). The numerical values used in the simulation have been chosen to have biological meaning (based on the references), as well as simulation convenience. The mortality rate, $\mu_{j, i}$, would also differ based on the type of cells it refers to. However, the values used in the simulations are the same for all cells. A more thorough discussion of this decision can be found in Section 6. The mutation probability is evenly distributed among the possible mutation outcomes. For example, a cell in class ( $j=3$ telomeres, $i=0$ mutations) can produce mutated daughters in classes $(j=3$ telomeres, $i=1$ mutation), ( $j=2$ telomeres, $i=1$ mutation), and ( $j=1$ telomere, $i=1$ mutation) with equal probability $\frac{1}{3} \times 10^{-5}$. The self-renewal probability of the cells refers to the asymmetric cell division assumption that one daughter of a cell will have the same characteristics (telomere length, in particular) as its mother. The remaining cell genealogy probabilities, referring to the daughter cell losing telomeres and not acquiring a mutation, are also evenly distributed among the possible outcomes.

\subsection{Linear Model Simulation}

The simulation for the linear model (Equation 3.4) represents an experiment of cellular dynamics with a relatively short duration. However, the main reason for the simulation is to present the different polynomial rates of growth for the different classes of cell population and to emphasize the fact that all cell types eventually develop, even though the initial cell population consists of only stem cells (which is implied in the result of Theorem 3.3.2). Only three telomere classes 
(corresponding to stem ( $j=3$ telomeres), progenitor $(j=2$ telomeres), and differentiated cells $(j=1$ telomere $)$ ) and only three mutation classes (normal cells $(i=0$ mutations), cells with one mutation ( $i=1$ mutation), and cancerous cells $(i=2$ mutations) $)$ are presented. Note that retinoblastoma is a cancer that develops after two mutations, which supports the assumption of the linear model simulation [27]. Initially, there are 10 million stem cells. Figures 3-6 show the different rates of polynomial growth of the different cell classes. In Figure 6, after 100 weeks we get the first cancer stem cell. At 200 weeks we have around 4 cancer stem cells and 170000 differentiated cancer cells. The ratio between the cancer stem cells and the differentiated cancer cells is consistent at that time with previous results [13].

\subsection{Nonlinear Model Simulation}

The nonlinear model simulation was run with $n=8$ telomere classes and $m=6$ mutation classes and presents a more complex example of a "Vogelgram" [22]. In other words, $j=8$ telomeres represents stem cells, $j=1$ telomere is the index of the telomere class of differentiated cells, $i=0$ mutations is the number of mutations for normal cells, and $i=5$ mutations is the index of the mutation class for cancer cells (i.e. cancer cells develop after acquiring 5 mutations). The crowding function $F$ is given by: $F(\vec{x})=0.0001\|\vec{x}\|_{1}, \vec{x} \in \mathbb{R}^{N}$, where $\|\cdot\|_{1}$ is the $l_{1}$ norm of the vector $\vec{x}$. The choice of $F$ describes loss of cells because the total cellular population is too large for the environment to handle because of spatial constraints. Therefore, cells stop proliferating or die and are not accounted for any further. One can view the scenario as cells entering a quiescent state. However, the numerical simulation is mainly used to portray the steady state equilibrium that is achieved in the nonlinear model, rather than depict a very specific phenomenon.

Different tissues have different stages of differentiation but it is known that erythropoiesis (production of red blood cells) has about 8 stages of differentiation [30]. Also, although different types of cancer go through a different number of mutations, 4-7 is the general number of mutations a cell needs to acquire to become cancerous [4][22]. The scenario we assumed for the nonlinear model is that the cells in a region of an organ, or in a cell culture dish, have reached homeostasis, consisting of only normal cells ( $i=0$ mutations). Once homeostasis is established, mutations were introduced and, hence, the possibility for cancer development. Homeostatic values were established at 0.0402333 million stem cells $(j=8$ telomeres) and 580.567 million differentiated cells $(j=1$ telomere). The full vector of values for the different differentiation classes of normal cells is: $\left.(580.567,49.3302,7.72654,1.68346,0.455944,0.14457,0.0517274,0.0402333)^{T}\right)$. Once mutations are introduced, only cancer cells ( $i=5$ mutations) remain after around 280 weeks (Figure 7). The resulting vector of the cancer cell populations is $(2690.99,244.64,40.7741,9.40954$, $2.68848,0.896172,0.336069,0.261392)^{T}$, where the first number refers to the number, in millions, of differentiated cancer cells ( $j=1$ telomere, $i=5$ mutations) and the last - the number, in millions, of cancer stem cells ( $j=8$ telomeres, $i=5$ mutations) in the region. The curve that shows the growth of the cancer cells is similar to the Gompertzian curve, which is achieved in many models [24][41][14]. The results, depending on the interpretation of the scenario, is that the cancer cells overtake either the region in the organ or the cell culture dish. 


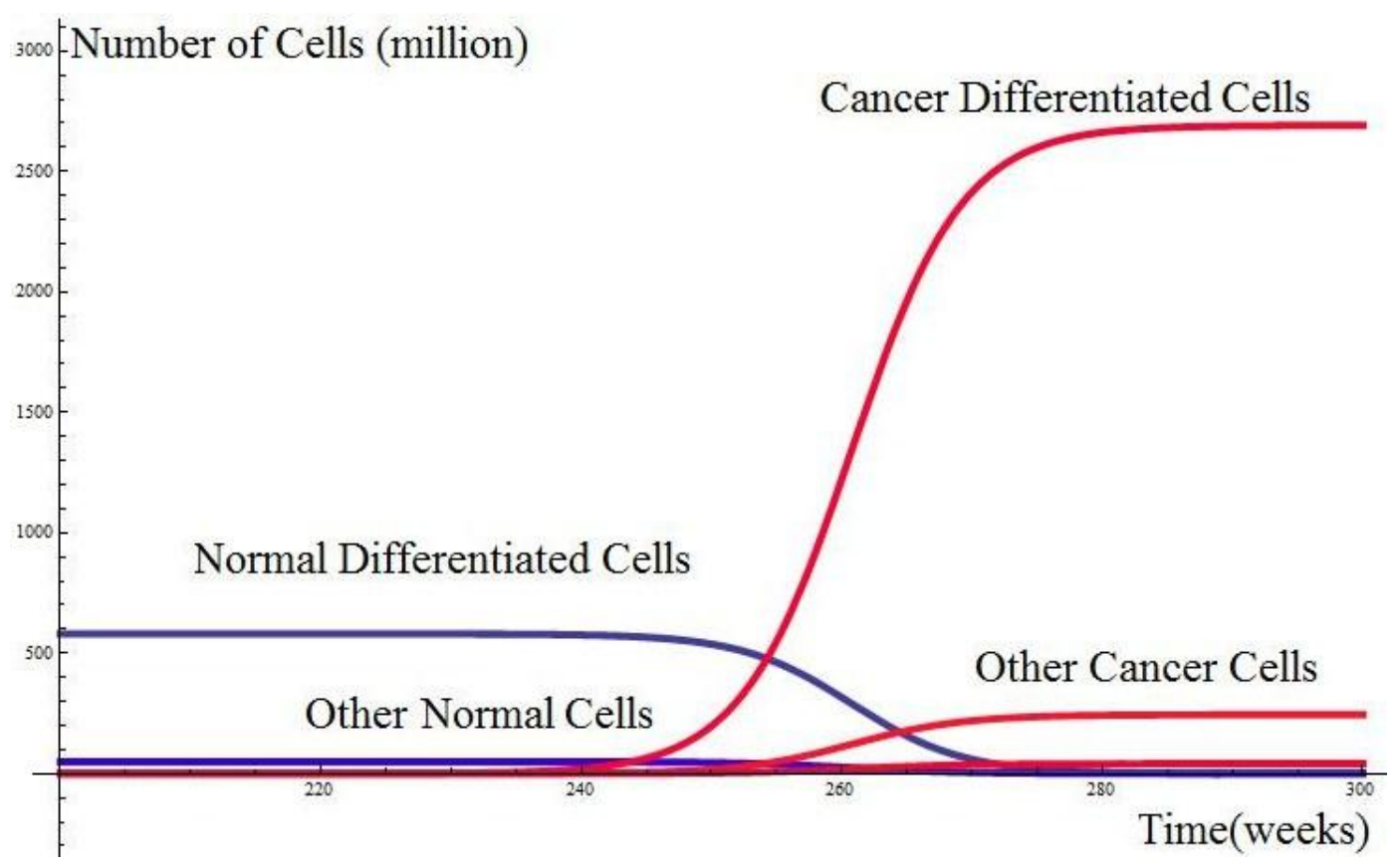

Figure 7: Nonlinear model with $n=8$ maximum number of telomeres and $m=6$ mutation classes (5 mutations necessary to reach malignancy). Cancer cells ( $i=5$ mutations) taking over the tissue environment according to the asymptotic steady state result in Theorem 4.3.

\section{Discussion}

This paper presents two models of intercellular dynamics: one linear and one nonlinear. Both models incorporate similar ideas: cells lose telomeres during mitosis and differentiation; cells can acquire mutations during division; when a normal cell acquires a certain number of mutations, it becomes cancerous[22][4].

In the Introduction, there were three questions we tried to address. Here are the answers, according to the presented models:

1. Question: Considering cell mutation as a dynamic population process, rather than a onetime random event, what can we show about cancer cell population growth in relation to the growth of the populations of non-cancer cells?

Answer: Theorem 3.3.2 proves that the number of cancer cells grows faster polynomially than any other type of cell (of the same telomere class). The Theorem does not assume any advantages of cancer cells in proliferation rates, mortality rates, and self-renewal probability. The only advantage cancer cells have over other cells, according to the model, is the number of mutations they have accumulated. According to Theorem 3.3.2, the more mutations a cell population has, the faster it grows with respect to time. Therefore, it is the nature of mutation acquisition that explains the higher population growth of cancer cells.

However, Theorem 3.3 does not address how quickly cancer develops and whether it devel- 
ops within a human life. This question was answered by the numerical simulations, in particular of the nonlinear scenario. In the simulation, equilibrium of normal cells was reached first, after which, with the given initial conditions, mutations were introduced and, hence, the possibility for the development of cancer cells. The experiments were run for several values of the proliferation rate of cancer cells. In our simulations, $\beta_{j, 5}=3.0$ cells $/$ week, $1 \leq j \leq 8$, gave the most realistic result as far as time-frame is concerned. From Figure 7 , one can see that cancer cells begin their invasion after about 240 weeks, become dominant after 250 weeks, and overtake the tissue (or cell dish) after 270 weeks. These results are consistent with the aggressive development of certain types of cancer, for example small cell lung cancer [36]. Therefore, according to the model and the current knowledge of the nature of cancer cells, cancer cells do need to exhibit high proliferation rate in order for their population to grow to levels dangerous for the organism in a realistic time frame.

2. Question: What is the role of stem cells in the cell population dynamics?

Answer: Theorem 3.3.2 proves that the coefficient of the highest power of the polynomial that determines the growth of the cell populations, relative to time, is a multiple of the initial number of stem cells. Therefore, stem cells are crucial for the development of all other cell classes and are also important for the rate at which those different cell populations grow. The importance of stem cells for the development of the somatic cells is known: [20][15][32][40]. The significance of cancer stem cells is shown through Theorem 4.3. The result does assume advantages for the cancer stem cell (higher proliferation rate, higher self-renewal probability), consistent with the characteristics associated with this type of cells [9][28] $[16][17][12][37][5][25]$. Under these assumptions, the cancer cells overtake the whole region of tissue (or cell culture dish), which confirms the importance of cancer stem cells for the aggressive development of cancer.

3. Question: Is the cancer stem cell count as small as scientists have claimed?

Answer: According to the numerical simulations, the ratio of cancer stem cells to overall cancer cell populations is quite small (order of 1 in 10000) and there has been evidence for similar ratios [13][9]. These specific results do depend on the different parameter values chosen for the simulations. However, mathematical evidence that cancer cells can be a small fraction of the total number cancer cells and still generate the cancer cell population can be found in Theorem 3.3.2, where it's shown that they grow at a slower polynomial rate than any other type of cancer cell, while differentiating into those types and, thus, constantly providing new cells for their lineage. Therefore, a relatively small subpopulation of cancer stem cells can generate the total population of cancer cells.

There are several assumptions of the basic framework of the model that should be commented on further. First, the model assumes that cancer stem cells mutate from normal stem cells. Although there is strong evidence for the existence of cancer cells that exhibit stem cell characteristics, their existence is still a hypothesis. Cancer stem cells have also been found only in certain types of cancer. Furthermore, the origin of cancer stem cells is ambiguous: some suggest they do come from mutated stem cells ([28]), others that they are mutated progenitor or differentiated cells. The 
model heavily relies on the assumption that stem cells mutate into cancer stem cells. In fact, it was designed to investigate the importance of this phenomenon and draw the conclusions above.

Second, Hypothesis 2.1 assumes asymmetric cell division (one daughter cell is identical with the mother cell and the other is genetically different). Differentiation of cells and loss of telomeres are well-established: [26][40][11]. However, the literature is not conclusive with the rate of telomere loss [29], which may be dynamic [23]. Further, the role of the enzyme telomerase has not been explicitly included in the model. Asymmetric division of stem cells has been demonstrated. In fact, one of the main properties of stem cells is self-renewal [40][32]. Also, it is hypothesized that some progenitor cells could self-renew as well [11]. Therefore, the assumption of the model that cells can self-renew is supportable. This assumption is only used for the analysis of the linear model and has no effect on the result of Theorem 4.3.

Third, Theorem 3.3.2 assumes $\mu_{j, i}=0$ for all cells and the nonlinear numerical simulation uses a constant mortality for all cell classes. For Theorem 3.3.2, this assumption is made so that the various rates of population growth of the different cell types can be observed. Therefore, the linear model with zero mortality is suitable for modeling short-term in vitro or in vivo experiments (mortality of cells would have less of an effect on the outcome of the experiment).

As far as the mortality rate used for the simulations goes, the focus of the model analysis is on the interaction between cell populations and, to some extent, the role of the proliferation rate in the inter-cellular dynamics. The mortality rate is, like the proliferation rate, dependent on the cell class. There exist mechanisms within the body that force mutated cells to go into apoptosis. Also, cancer cells often have lower mortality rate than normal cells [25]. One can explore those options by manipulating the mortality rate for certain cell classes. However, such investigation is beyond the scope of this paper.

Finally, the assumption that cells acquire their sequence of mutation in a particular order may be too simplistic [42]. However, the model does not address different types of mutation, only their number. So, the focus is on the process of mutation acquisition and not on the effect of specific mutations on the cell characteristics.

The linear model with zero mortality can be used to model short term in vitro and in vivo experiments: cell mortality does not play a major role in a short time span. Linear and polynomial rates of growth of cancer cell populations have been observed: [21][15][37][12][10][38]. Further, polynomial growth of high degree is similar in behavior to exponential growth if the time frame is short, and exponential models are often used to describe initial populations growth.

The numerical simulation of the nonlinear model shows growth of the cancer cell population similar to the Gompertzian curve, which is achieved in many models [24][41][14]. This result supports the usefulness of the model framework for modeling both in-vivo and in-vitro experiments by showing its consistency with previous work.

The strength of the modeling framework presented in the current paper lies within its flexibility. The linear model can predict short-term behavior, the nonlinear - long term behavior. Further, the choice of the operator $F$ is up to the researcher and allows for the investigation of other questions within the intra-cellular dynamics. More, we have made suggestions for future researchers and how they can address different problems by simply modifying the parameters of the model. Those suggestions and different scenarios will be investigated in future work. Future work would 
also feature the addition of fitness and competition between healthy and cancer cells, investigating possible cellular Darwinism. Another flexibility of the model is the open number of telomere and mutation classes, which implies that one can mimic different tissues and different types of cancer. Therefore, the presented models, although complex, can be readily used to describe a wide variety of cell interactions, which makes the model framework useful for researchers.

\section{Acknowledgements}

The author would like to thank prof. Glenn Webb for his patience and constant support throughout the writing process and prof. Joanna Wares for introducing him into the world of Biomathematics.

\section{References}

[1] S. Ahmed, J.F. Passos, M.J. Birket, T. Beckmann, S. Brings, H. Peters, M.A. Birch-Machin, T. von Zglinicki, G. Saretzki. Telomerase Does Not Counteract Telomere Shortening But Protects Mitochondrial Function Under Oxidative Stress. Journal of Cell Science, 121 (2008), No. 7, 1046-1053.

[2] O. Arino, M. Kimmel, G.F. Webb. Mathematical Modeling of the Loss of Telomere Sequences. J. theor. Biol., 177 (1995), No. 1, 45-57.

[3] O. Arino, E. Sánchez, G.F. Webb. Polynomial Growth Dynamics of Telomere Loss in a Heterogeneous Cell Population. Dynamic Control Discrete Impulsive System, 3 (1997), No. 3 , 263-282.

[4] P. Armitage, R. Doll. The age distribution of cancer and a multi-stage theory of carcinogenosis. IJE, 33 (2004), No. 6, 1174-1179.

[5] S. Bagheri, M. Nosrati, S. Li, S. Fong, S. Torabian, J. Rangel, D.H. Moore, S. Federman, R.R. LaPosa, F.L. Baehner, R.W. Sagebiel, J.E. Cleaver, C. Haqq, R.J. Debs, E.H. Blackburn, M. Kashani-Sabet. Genes and pathways downstream of telomerase in melanoma metastasis. PNAS, 103 (2006), No. 30, 11306-11311.

[6] H.T. Banks, K.L. Sutton, W.C. Thompson, G. Bocharov, D. Roose, T. Schenkel, A. Meyerhans. Estimation of Cell Proliferation Dynamics Using CFSE Data. Bulletin of Mathematical Biology, 73 (2011), No. 1, 116-150.

[7] S. Bernard, L. Pujo-Menjouet, M.C. Mackey. Analysis of Cell Kinetics Using a Cell Division Marker: Mathematical Modeling of Experimental Data. Biophysical Journal, 84 (2003), No. 5, 3414-3424.

[8] D.S. Bernstein. Matrix Mathematics, Second Edition., Princeton University Press, 2009. 
[9] D. Bonnet, J.E. Dick. Human Acute Myeloid Leukemia is Organized as a Hierarchy That Originates From a Primitive Hematopoetic Cell. Nature Medicine, 3 (1997), No. 7, 730-737.

[10] A. Brú, S. Albertos, J.L. Subiza, J.L. García-Asenjo, I. Brú. The Universal Dynamics of Tumor Growth. Biophysical Journal, 85 (2003), No. 5, 2948-2961.

[11] E.D. Cohen, Y. Tian, E.E. Morrisey. Wnt signaling: an essential regulator of cardiovascular differentiation, morphogenesis and progenitor self-renewal. Development, 135 (2008), No. 5, 789-798.

[12] A.T. Collins, P.A. Berry, C. Hyde, M.J. Stower, N.J. Maitland. Prospective Identification of Tumorigenic Prostate Cancer Stem Cells. Cancer Res., 65 (2005), No. 23, 10946-10951.

[13] P. Dalerba, S.J. Dylla, I.-K. Park, R. Liu, X. Wang, R.W. Cho, T. Hoey, A. Gurney, E.H. Huang, D.M. Simeone, A.A. Shelton, G. Parmiani, C. Castelli, M.F. Clarke. Phenotypic characterization of human colorectal cancer stem cells. PNAS, 104 (2007), No. 24, 10158-10163.

[14] L.G. de Pillis, A.E. Radunskaya, C.L. Wiseman. A Validated Mathematical Model of CellMediated Immune Response to Tumor Growth. Cancer Res., 65 (2005), No. 17, 7950-7958.

[15] B.M. Deasy, R.J. Jankowski, T.R. Payne, B. Cao, J.P. Goff, J.S. Greenberger, J. Huard. Modeling Stem Cell Population Growth: Incorporating Terms for Proliferative Heterogeneity. Stem Cells, 21 (2003), No. 5, 536 - 545.

[16] J.E. Dick. Breast cancer stem cells revealed. PNAS, 100 (2003), No. 7, 3547-3549.

[17] D. Dingli, F. Michor. Successful Therapy Must Eradicate Cancer Stem Cells. Stem Cells, 24 (2006), No. 12, 2603-2610.

[18] J. Dyson, E. Sánchez, R. Villella-Bressan, G.F. Webb. Stabilization of telomeres in nonlinear models of proliferating cell lines. Journal of Theoretical Biology, 244 (2007), No. 3, 400-408.

[19] J. Dyson, R. Villella-Bressan, G.F. Webb. Asymptotic Behaviour Of Solutions To Abstract Logistic Equations. Mathematical Biosciences, 206 (2007), No. 2, 216-232.

[20] H. Enderling, D. Park, L. Hlatky, P. Hahnfeldt. The Importance of Spatial Distribution of Stemness and Proliferation State in Determining Tumor Radioresponse. Math. Model. Nat. Phenom., 4 (2009), No. 3, 117-133.

[21] A. Eramo, F. Lotti, G. Sette, E. Pilozzi, M. Biffoni, A. Di Virgilio, C. Conticello, L. Ruco, C. Peschle, R. De Maria. Identification and expansion of the tumorigenic lung cancer stem cell population. Cell Death and Differentiation, 15 (2008), No. 3, 504-514.

[22] E.R. Fearon, B. Vogelstein. A Genetic Model for Colorectal Tumorigenesis. Cell, 61 (1990), 759-767. 
[23] R.W. Frenck, Jr., E.H Blackburn, K.M. Shannon. The rate of telomere sequence loss in human leukocytes varies with age. PNAS, 95 (1998), No. 10, 5607-5610.

[24] S.N. Gentry, R. Ashkenazi, T.L. Jackson. A Maturity Structured Mathematical Model of Mutation Acquisition in the Absence of Homeostatic Regulation. Math. Model. Nat. Phenom., 4 (2009), 403-422.

[25] D. Hanahan, R.A. Weinberg. The Hallmarks of Cancer: The Next Generation. Cell., 144 (2011), No. 5, 646-674.

[26] K.E. Huffman, S.D. Levene, V.M. Tesmer, J.W. Shay, W.E. Wright. Telomere Shortening Is Proportional to the Size of the G-rich Telomeric 3'-Overhang. The Journal of Biological Chemistry, 275 (2000), No. 26, 19719-19722.

[27] A. G. Knudson Two genetic hits (more or less) to cancer. Nat. Rev. Cancer., 1 (2001), 157162.

[28] S.H. Lang, F.M. Frame, A.T. Collins. Prostate cancer stem cells. Journal of Pathology, 217 (2009), No. 9, 299-306.

[29] M.Z. Levy, R.C. Allsopp, A.B. Futcher, C.W. Greider, C.B. Harley. Telomere End-replication Problem and Cell Aging. J. Mol. Biol., 225 (1992), No. 4, 951-960.

[30] H. Lodish, J. Flygare, S. Chou. From stem cell to erythroblast: Regulation of red cell production at multiple levels by multiple hormones. IUBMB Life, 62 (2010), No. 7, 492-496.

[31] A. Marciniak-Czochra. Mathematical models of stem cells renewal and differentiation. Oberwolfach Reports, 2 (2009), 3414-3424.

[32] S.J. Morrison, N. Uchida, I.L. Weissman. The biology of hematopoietic stem cells. Annu. Rev. Cell Dev. Biol., 11 (1995), 35-71.

[33] P. Olofsson. Modeling of the Process of Telomere Shortening: an Overview.

[34] L. Perko. Differential Equations and Dynamical Systems, $3^{\text {rd }}$ edition. Springer, New York, NY, 2001.

[35] F. Roegiers, Y.N. Jan. Asymmetric cell division. Current Opinion in Cell Biology, 16 (2004), No. 2, 195-205.

[36] G.R. Simon, H. Wagner. Small Cell Lung Cancer*. Chest, 123 (2003), No. 1, 259-271.

[37] S.K. Singh, I.D. Clarke, M. Terasaki, V.E. Bonn, C. Hawkins, J. Squire, P.B. Dirks. Identification of a Cancer Stem Cell in Human Brain Tumors. Cancer Res., 63 (2003), No. 18, 5821-5828.

[38] P. Skehan, S.J. Friedman. Non-exponential growth by mammalian cells in culture. Cell Tissue Kinet., 17 (1984), No. 4, 335-343. 
[39] G.I. Solyanik, N.M. Berezetskaya, R.I. Bulkiewicz, G.I. Kulik. Different growth patterns of a cancer cell population as a function of its starting growth characteristics: analysis by mathematical modelling. Cell Prolif, 28 (1995), No. 5, 263-278.

[40] G.J. Spangrude, S. Heimfeld, I.L. Weissman. Purification and characterization of mouse hematopoietic stem cells. Science, 244 (1988), No. 4861, 58-62.

[41] J.F. Speer, V.E. Petrosky, M.W. Retsky, R.H. Wardwell", A Stochastic Numerical Model of Breast Cancer Growth That Simulates Clinical Data. Cancer Res., 44 (1984), No. 9, 41244130 .

[42] K. Sprouffske, J.W. Pepper, C.C. Maley. Accurate Reconstruction of the Temporal Order of Mutations in Neoplastic Progression. Cancer Prev. Res., 4 (2011), No. 7, 1135-1144.

[43] S.A. Stewart, W.C. Hahn, B.F. O'Connor, E.N. Banner, A.S. Lundberg, P. Modha, H. Mizuno, M.W. Brooks, M. Fleming, D.B. Zimonjic, N.C. Popescu, R.A. Weinberg. Telomerase contributes to tumorigenesis by a telomere length-independent mechanism. PNAS, 99 (2002), No. 20, 12606-12611.

[44] M.R. Stratton, P.J. Campbell, P.A. Futreal. The Cancer Genome. Nature, 458 (2009), No. 7239, 156-182.

[45] L.G. van der Flier, H. Clevers. Stem Cells, Self-Renewal, and Differentiation in the Intestinal Epithelium. Annu. Rev. Physiol., 71 (2009), No. 1, 241-260.

[46] T. von Zglinicki. Oxidative Stress Shortens Telomeres. Trends in Biochemical Scoences, 27 (2002), No. 7, 339-344.

[47] G.F. Webb. Logistic Models Of Structured Population Growth. Comp and Maths. with Appls., 12 (1986), No. 4-5A, 527-539.

[48] G.D. Weinstein, J.L. McCullough, P. Ross. Cell Proliferation in Normal Epidermis. The Journal of Investigative Dermatology, 82 (1984), No. 6, 623-628.

[49] G.D. Wilson, N.J. McNally, S. Dische, M.I. Saunders, C. Des Rochers, A.A. Lewis, M.H. Bennett. Measurement of cell kinetics in human tumours in vivo using bromodeoxyuridine incorporation and flow cytometry. Br. J. Cancer, 58 (1988), No. 4, 423-431.

[50] Q.-L. Ying, J. Wray, J. Nichols, L. Batlle-Morera, B. Doble, J. Woodgett, P. Cohen, A. Smith. The ground state of embryonic stem cell self-renewal. Nature, 453 (2008), No. 7194, 519523. 


\section{Appendix}

Claim: Let $C$ and $B$ be $n \times n$ non-zero upper-triangular matrices. Let the first $k$ superdiagonals of $C$ (the main diagonal is also considered a superdiagonal) be zero and the first $m$ superdiagonals of $B$ be zero, $0 \leq k, m \leq n-1$. Assume every element on the $l^{\text {th }}$ superdiagonal of $C$, adopting the indexing from above, $l \geq k$, and $r^{t h}$ superdiagonal of $B, r \geq m$ is positive. Then $C B$ and $B C$ are upper-triangular matrices in which exactly the first $k+m$ superdiagonals are zero (if $k+m \geq n$, $C B$ and $B C$ will be the zero matrix) and every element of the $s^{t h}$ superdiagonal, $s \geq k+m$ is positive.

In other words, if:

$$
C=\left(\begin{array}{lllll}
0 & 0 & 0 & 1 & 1 \\
0 & 0 & 0 & 0 & 1 \\
0 & 0 & 0 & 0 & 0 \\
0 & 0 & 0 & 0 & 0 \\
0 & 0 & 0 & 0 & 0
\end{array}\right)
$$

(The first 3 superdiagonals of $C$ are 0 ),

$$
B=\left(\begin{array}{lllll}
0 & 1 & 1 & 1 & 1 \\
0 & 0 & 1 & 1 & 1 \\
0 & 0 & 0 & 1 & 1 \\
0 & 0 & 0 & 0 & 1 \\
0 & 0 & 0 & 0 & 0
\end{array}\right)
$$

(The first superdiagonal of $B$ is 0 ), then

$$
B C=C B=\left(\begin{array}{ccccc}
0 & 0 & 0 & 0 & 1 \\
0 & 0 & 0 & 0 & 0 \\
0 & 0 & 0 & 0 & 0 \\
0 & 0 & 0 & 0 & 0 \\
0 & 0 & 0 & 0 & 0
\end{array}\right)
$$

(The first $3+1=4$ superdiagonals of $B C$ and $C B$ are 0 ).

Lemma 6.1. 3.5 If $1 \leq k \leq m-1$, the $k^{\text {th }}$ block subdiagonal is the highest indexed positive block subdiagonal of $A^{k}$. Any block subdiagonal of higher index is 0 .

Proof.(By induction) Basic Step: In A, by construction, the highest indexed positive block subdiagonal is its first block subdiagonal.

Inductive Step: Let the statement be true for $A^{k}, 1 \leq k \leq m-2$. Pick an arbitrary block element in the $(k+1)^{t h}$ block subdiagonal of $A^{k+1}, A_{l+k+1, l}^{k+1}, 1 \leq l \leq m-k-2$. It is constructed by matrix multiplying the $(l+k+1)^{t h}$ block row of $A^{k}$ with the $l^{\text {th }}$ block-column of $A$. From the structure of $A, A_{l+k+1, l}^{k+1}=A_{l+k+1, l}^{k} P_{l-1}+A_{l+1}^{l+k+1} Q_{l}$. From the inductive assumption, $A_{l+k+1, l}^{k}=0$, since it is an element in the $(k+1)^{t h}$ block subdiagonal of $A^{k}$. However, $A_{l+k+1, l+1}^{k}$, which is an 
element in the $k^{\text {th }}$ block subdiagonal of $A^{k}$, is positive. From the Claim and the fact that $Q_{l}$ has no zero superdiagonals, $A_{l+1}^{l+k+1} Q_{l} \neq 0$. Therefore, the $(k+1)^{t h}$ block subdiagonal of $A^{k+1}$ is positive. Consider the $(k+2)^{t h}$ subdiagonal of $A^{k+1}$. If $k=m-2$, it does not exist. So, let $k<m-2$. Let $A_{l+k+2, l}^{k+1}, 1 \leq l \leq$ be an arbitrary element in the $(k+2)^{t h}$ subdiagonal of $A^{k+1}$. By the rules of matrix multiplication, and the structure of $A, A_{l+k+2, l}^{k+1}=A_{l+k+2, l}^{k} P_{l-1}+A_{l+k+2, l+1}^{k} Q_{l}$. However, $A_{l+k+2, l}^{k}$ is an element in the $(k+2)^{t h}$ block subdiagonal of $A^{k}$ and $A_{l+k+2, l+1}^{k}$ is an element in the $(k+1)^{t h}$ block subdiagonal of $A^{k}$. From the inductive assumption, both the $(k+1)^{t h}$ and the $(k+2)^{t h}$ block subdiagonals of $A^{k}$ are 0 . Therefore, the $(k+2)^{t h}$ subdiagonal of $A^{k+1}$ is 0 , which proves the Lemma.

Lemma 6.2. 3.6 In $A^{k}, 1 \leq k \leq m+n$, any block element of a positive block subdiagonal indexed by $i, 0 \leq i \leq m-1$, is an upper-triangular matrix in which exactly the first $k-i$ superdiagonals (including the main diagonal) are 0.

Proof.(By induction) Basic Step: In $A$, the only positive block subdiagonals are the $0^{\text {th }}$ and the $1^{\text {st }}$. An arbitrary element of the $0^{t h}$ block subdiagonal of $A$ is $P_{i-1}, 1 \leq i \leq m$, which is an uppertriangular matrix whose $0^{\text {th }}$ superdiagonal is 0 and the rest are positive. An arbitrary element of the $1^{\text {th }}$ block subdiagonal of $A$ is $Q_{i}, 1 \leq i \leq m$, which is an upper-triangular matrix without zero superdiagonals.

Inductive step: Let the statement be true for $A^{k}, 1 \leq k \leq m+n-1$. Assume $A^{k+1}$ has a positive block subdiagonal whose index is $i, 0 \leq i \leq m-1$. Pick and arbitrary block element of its block subdiagonal $A_{l+i, l}^{k+1}, 1 \leq l \leq m-i$. It is constructed by matrix multiplying the $(l+i)^{t h}$ block row of $A^{k}$ with the $l^{\text {th }}$ block-column of $A$. The only positive block elements of the $l^{\text {th }}$ blockcolumn of $A$ are $A_{l, l}=P_{l-1}$ and $A_{l+1, l}=Q_{l}$. Therefore, by the rules of matrix multiplication, $A_{l+i, l}^{k+1}=A_{l+i, l}^{k} P_{l-1}+A_{l+i, l+1}^{k} Q_{l}$.

Case 1: Let $A_{l+i, l}^{k}=0$. Then $A_{l+i, l+1}^{k} \neq 0$, by assumption. It is a block element in the $(i-1)^{t h}$ block subdiagonal of $A^{k}$, so it is an upper-triangular matrix in which exactly the first $k-i+1$ superdiagonals are 0 . From the Claim and $Q_{l}$ being an upper-triangular matrix without 0 superdiagonals, $A_{l+i, l}^{k+1}$ is an upper-triangular matrix for which exactly the first $k-i+1$ superdiagonals are 0 .

Case 2: Let $A_{l+i, l+1}^{k}=0$. Then, by assumption, $A_{l+i, l}^{k}$ is a positive block element in the $i^{\text {th }}$ block subdiagonal of $A^{k}$, so it is an upper-triangular matrix in which exactly the first $k-i$ superdiagonals are 0 . From the Claim and $P_{l-1}$ being an upper-triangular matrix whose only 0 superdiagonal is its $0^{t h}, A_{l+i, l}^{k+1}$ is an upper-triangular matrix in which exactly the first $k-i+1$ superdiagonals are 0 .

Case 3: $A_{l+i, l}^{k} \neq 0$ and $A_{l+i, l+1}^{k} \neq 0$. Then, from Cases 1 and $2, A_{l+i, l}^{k+1}$ is the sum of two upper-triangular matrices in which exactly the first $k-i+1$ superdiagonals are 0 . Hence, $A_{l+i, l}^{k+1}$ is an upper-triangular matrix in which exactly the first $k-i+1$ superdiagonals are 0 .

Lemma 6.3. 3.7 If $n \leq k<n+m$, the $(k-n+1)^{\text {th }}$ block subdiagonal is the lowest indexed positive block subdiagonal of $A^{k}$. Any block subdiagonal of lower index is 0 .

Proof.(By induction) Basic Step: Since $A$ is a lower-triangular block matrix, the main block diagonal block elements of $A^{n}, A_{i, i}^{n}=P_{i-1}^{n}, 1 \leq i \leq m$. From the Claim, since the main diagonal 
of $P_{i-1}$ is $0, P_{i-1}^{n}=0$. From Lemma 3.6, the first block subdiagonal of $A^{n}$ consists of uppertriangular matrices, in which exactly the first $n-1$ superdiagonals are 0 . Hence, the first block subdiagonal of $A^{n}$ is positive.

Inductive step: Assume the statement is true for $A^{k}, n<k<n+m$. Then, from Lemma 3.6, the $(k-n+2)^{t h}$ block subdiagonal of $A^{k}$ consists of upper-triangular matrices in which exactly the first $n-2$ superdiagonals are 0 and the $(k-n+1)^{t h}$ block subdiagonal of $A^{k}$ consists of uppertriangular matrices in which exactly the first $n-1$ superdiagonals are 0 . Consider $A^{k+1}$. Pick and arbitrary block element in its $(k-n+2)^{t h}$ block subdiagonal, $A_{l+k-n+2, l}^{k+1}, 1 \leq l \leq m-k+n-2$. It is constructed by matrix multiplying the $(l+k-n+2)^{t h}$ block row of $A^{k}$ with the $l^{\text {th }}$ block-column of A. The only positive block elements of the $l^{\text {th }}$ block-column of A are $A_{l, l}=P_{l-1}$ and $A_{l+1, l}=Q_{l}$. Therefore, by the rules of matrix multiplication, $A_{l+k-n+2, l}^{k+1}=A_{l+k-n+2, l}^{k} P_{l-1}+A_{l+k-n+2, l+1}^{k} Q_{l}$. $A_{l+k-n+2, l+1}^{k}$ is a block element in the $(k-n+1)^{t h}$ block subdiagonal of $A^{k}$ and $Q_{l}$ is an uppertriangular matrix without 0 superdiagonals. Therefore, by the Claim, the result of their matrix multiplication is an upper-triangular matrix in which exactly the first $n-1$ superdiagonals are 0 . $A_{l+k-n+2, l}^{k}$ is a block element in the $(k-n+2)^{t h}$ block subdiagonal of $A^{k}$ and $P_{l-1}$ is an uppertriangular matrix whose main diagonal is 0 . Therefore, by the Claim, the result of their matrix multiplication is an upper-triangular matrix in which exactly the first $n-1$ superdiagonals are 0 . Hence, $A_{l+k-n+2, l}^{k+1}$ is an upper-triangular matrix in which exactly the first $n-1$ superdiagonals are 0 and the last one is positive. Pick and arbitrary block element in the $(k-n+1)^{t h}$ block subdiagonal of $A^{k+1}, A_{l+k-n+1, l}^{k+1}, 1 \leq l \leq m-k+n-1$. It is constructed by matrix multiplying the $(l+k-n+1)^{t h}$ block row of $A^{k}$ with the $l^{t h}$ block-column of A. The only positive block elements of the $l^{\text {th }}$ block-column of $\mathrm{A}$ are $A_{l, l}=P_{l-1}$ and $A_{l+1, l}=Q_{l}$. Therefore, by the rules of matrix multiplication, $A_{l+k-n+1, l}^{k+1}=A_{l+k-n+1, l}^{k} P_{l-1}+A_{l+k-n+1, l+1}^{k} Q_{l}$. $A_{l+k-n+1, l+1}^{k}$ is a block element in the $(k-n)^{t h}$ block subdiagonal of $A^{k}$, so it is $0 . A_{l+k-n+1, l}^{k}$ is a block element in the $(k-n+1)^{t h}$ block subdiagonal of $A^{k}$ and $P_{l-1}$ is an upper-triangular matrix whose main diagonal is 0 . Therefore, by the Claim, the result of their matrix multiplication is 0 . Hence, $A_{l+k-n+1, l}^{k+1}$ is 0 . Similar results would follow for any other lower indexed subdiagonal of $A^{k+1}$. 